\title{
Plasticity of the $\beta$-Trefoil Protein Fold in the Recognition and Control of Invertebrate Predators and Parasites by a Fungal Defence System
}

\author{
Mario Schubert ${ }^{19}$, Silvia Bleuler-Martinez ${ }^{29}$, Alex Butschi ${ }^{3}$, Martin A. Wälti ${ }^{2}$, Pascal Egloff ${ }^{2}$, Katrin Stutz ${ }^{3}$, \\ Shi Yan ${ }^{4}$, lain B. H. Wilson ${ }^{4}$, Michael O. Hengartner ${ }^{3}$, Markus Aebi ${ }^{2}$, Frédéric H.-T. Allain ${ }^{1 *}$, \\ Markus Künzler ${ }^{2 *}$
}

1 Institute of Molecular Biology and Biophysics, ETH Zürich, Zürich, Switzerland, 2 Institute of Microbiology, ETH Zürich, Zürich, Switzerland, 3 Institute of Molecular Life Sciences, University of Zürich, Switzerland, 4 Department of Chemistry, University of Natural Resources and Life Sciences (BOKU), Vienna, Austria

\begin{abstract}
Discrimination between self and non-self is a prerequisite for any defence mechanism; in innate defence, this discrimination is often mediated by lectins recognizing non-self carbohydrate structures and so relies on an arsenal of host lectins with different specificities towards target organism carbohydrate structures. Recently, cytoplasmic lectins isolated from fungal fruiting bodies have been shown to play a role in the defence of multicellular fungi against predators and parasites. Here, we present a novel fruiting body lectin, CCL2, from the ink cap mushroom Coprinopsis cinerea. We demonstrate the toxicity of the lectin towards Caenorhabditis elegans and Drosophila melanogaster and present its NMR solution structure in complex with the trisaccharide, GlcNAc $\beta 1,4[\mathrm{Fuc} \alpha 1,3] \mathrm{GlcNAc}$, to which it binds with high specificity and affinity in vitro. The structure reveals that the monomeric CCL2 adopts a $\beta$-trefoil fold and recognizes the trisaccharide by a single, topologically novel carbohydratebinding site. Site-directed mutagenesis of CCL2 and identification of $C$. elegans mutants resistant to this lectin show that its nematotoxicity is mediated by binding to $\alpha 1,3$-fucosylated N-glycan core structures of nematode glycoproteins; feeding with fluorescently labeled CCL2 demonstrates that these target glycoproteins localize to the $C$. elegans intestine. Since the identified glycoepitope is characteristic for invertebrates but absent from fungi, our data show that the defence function of fruiting body lectins is based on the specific recognition of non-self carbohydrate structures. The trisaccharide specifically recognized by CCL2 is a key carbohydrate determinant of pollen and insect venom allergens implying this particular glycoepitope is targeted by both fungal defence and mammalian immune systems. In summary, our results demonstrate how the plasticity of a common protein fold can contribute to the recognition and control of antagonists by an innate defence mechanism, whereby the monovalency of the lectin for its ligand implies a novel mechanism of lectin-mediated toxicity.
\end{abstract}

Citation: Schubert M, Bleuler-Martinez S, Butschi A, Wälti MA, Egloff P, et al. (2012) Plasticity of the $\beta$-Trefoil Protein Fold in the Recognition and Control of Invertebrate Predators and Parasites by a Fungal Defence System. PLoS Pathog 8(5): e1002706. doi:10.1371/journal.ppat.1002706

Editor: Stuart M. Levitz, University of Massachusetts Medical School, United States of America

Received January 16, 2012; Accepted April 2, 2012; Published May 17, 2012

Copyright: (C) 2012 Schubert et al. This is an open-access article distributed under the terms of the Creative Commons Attribution License, which permits unrestricted use, distribution, and reproduction in any medium, provided the original author and source are credited.

Funding: This work was supported by the NIGMS (grant no. GM62116 to the Consortium for Functional Glycomics), the European Commission's Marie Curie program (EuroGlycoArrays ITN), the Swiss National Science Foundation (grant no. 31003A-130671 to MK, MOH and MA, SNF-NCCR Structural Biology to FH-TA, SNF Sinergia grant CRSII3_127333 to FH-TA and MA) and ETH Zürich. The funders had no role in study design, data collection and analysis, decision to publish, or preparation of the manuscript.

Competing Interests: The authors have declared that no competing interests exist.

*E-mail: markus.kuenzler@micro.biol.eth (MK); allain@mol.biol.ethz.ch (FHTA)

9 These authors contributed equally to this work.

\section{Introduction}

Adequate and efficient defence mechanisms to protect an organism's integrity and survival have been essential for the evolution of multicellularity since loss of individual cells may be detrimental for a multicellular organism. Any defence mechanism thereby critically relies on the ability to discriminate between self and non-self. Since all living cells display specific carbohydrate structures on their surface [1], glycans have been used for the recognition of non-self since the beginning of multicellular life [2]. Accordingly, many of the proteins that are able bind to specific carbohydrate structures, commonly referred to as lectins, have been implicated in defence, mainly in the innate immune systems of animals which is considered an ancestral defence mechanism and a first and immediate line of defence against potentially harmful microorganisms [3]. These lectins are either membranebound or secreted and localize to the interface between the host and the environment where they bind to microorganismassociated carbohydrates and function either as receptors triggering the expression of host immune effectors, by opsonizing the microorganisms for host immune effectors or immune cells (reviewed in [4]) or as direct immune effectors by killing the microorganism upon binding [5-8]. In analogy to latter function of combining non-self recognition and killing, plants use insecticidal lectins to defend themselves against herbivorous insects [9]. Recently, a group of fungal lectins, commonly referred to as fruiting body lectins, has been shown to play a role in the defence of multicellular fungi against predators and parasites based on their toxicity to various model organisms [10-15]. According to the above role of lectins in defence, most defence lectins should be 


\section{Author Summary}

All multicellular organisms have developed mechanisms to defend themselves against predators, parasites and pathogens. As a common mechanism, animals, plants and fungi use a large arsenal of carbohydrate-binding proteins (lectins) to protect themselves from predation and parasitism. The success of this type of innate defence mechanism critically depends on the diversity of specific recognition of foreign carbohydrate structures by the host lectins. In this study, we use NMR structure determination to show that part of this diversity is created by the plasticity of common protein folds. The identified fungal lectin that is toxic to nematodes and insects, adopts a common lectin fold but is remarkable in terms of its specificity and affinity for the recognized foreign carbohydrate structure, the number and location of the carbohydrate binding sites on the protein and the degree of oligomerization. Since the identified in vivo target of the fungal lectin is characteristic for invertebrates, our results may be exploited to develop novel approaches for the control of animal and human parasites.

specific for carbohydrate structures that do not exist in the host (are non-self) and are characteristic for the target organism. To date, only very few target carbohydrate structures or glycoconjugates of such lectins involved in innate defence mechanisms have been identified and their recognition by the lectin investigated at molecular level $[5,8,10,16,17]$.

In organisms lacking an antibody-based adaptive immunity, such a lectin-based defence strategy critically relies on a large diversity in carbohydrate specificities. This diversity can be achieved either by diversification on the level of lectin folds and/or by the plasticity of a common lectin fold. The known fruiting body lectins belong to six structural families [14] of which the $\beta$-propeller-fold lectins, actinoporin-like lectins, galectins and $\beta$-trefoil (ricin B or R-type) lectins [18] are the most prominent ones. Some of these lectins are multidomain proteins harbouring in addition a cysteine protease/dimerization domain (R-type Marasmius oreades agglutinin [MOA] and Polyporus squamosus lectin [PSL]) $[19,20]$ or a pore-forming module (R-type Laetiporus sulphureus lectin [LSL]) [21]. In the first case, it was demonstrated that both domains are required for toxicity [10] suggesting that the lectin domain guides the catalytic domain to specific target structures. However, most lectins implicated in the defence of plants and fungi are composed just of lectin domains and contain multiple binding sites for either the same or different carbohydrate structures. For some of these lectins it has been demonstrated that this multivalency is essential for their toxicity [22]. These results suggest that lectin-mediated toxicity involves crosslinking of glycoconjugates but the exact mechanism remains unclear.

We describe the identification and characterization of a novel, monovalent lectin, CCL2, from fruiting bodies of the ink cap mushroom Coprinopsis cinerea and present the NMR structure of CCL2 in ligand-free form and in complex with its in vivo ligand. The lectin was found to bind specifically and with an atypical high affinity to Fuc $\alpha 1,3$-modified core $\mathrm{N}$-glycans in vitro, using a single, topologically novel binding site on its $\beta$-trefoil fold. N-glycans carrying such a modification are characteristic for invertebrates but absent from fungi. We applied biotoxicity assays to demonstrate toxicity towards two model invertebrates. In accordance with the in vitro binding data, the nematotoxicity of CCL2 was dependent on core $\alpha 1,3$-fucosylation of $C$. elegans $\mathrm{N}$-glycans on intestinal proteins of the nematode. These results show how multicellular organisms exploit the plasticity of a common protein fold to create a novel lectin specificity and an alternative mechanism of lectin-mediated toxicity for defence.

\section{Results}

\section{Identification, cloning and expression of CCL2 from Coprinopsis cinerea}

We detected a soluble $15 \mathrm{kDa}$ protein from fruiting bodies of the model mushroom $C$. cinerea by virtue of its binding to horseradish peroxidase (HRP) in immunoblots. The protein was present in extracts from fruiting bodies but not from vegetative mycelium, indicating a fruiting body-specific expression. We isolated the protein using HRP-affinity chromatography (Figure 1A) and identified it as hypothetical protein CC1G_11781 of C. cinerea strain Okayama7 by MALDI-MS/ MS. Since the protein, termed CCL2 (Coprinopsis cinerea lectin 2), was extracted from fruiting bodies of the C. cinerea strain AmutBmut (Swamy et al 1984), the respective genomic locus of strain AmutBmut was cloned and sequenced. This sequence served as a basis for the cloning of the respective cDNA from total RNA isolated from AmutBmut fruiting bodies. A second cDNA, coding for an isoprotein ( $52 \%$ identity; Table S1), termed CGL1 (Coprinopsis cinerea lectin 1) (CG1G_11778), was cloned and sequenced accordingly. The two proteins are predicted to contain neither a signal sequence for classical secretion nor $\mathrm{N}$-glycosylation sites. The cDNAs coding for CCL1 and CCL2 were cloned in pET expression vectors and the proteins were expressed in the cytoplasm of $E$. coli BL21(DE3). The recombinant proteins were highly expressed and soluble (Figure $\mathrm{S} 1$ ) and versions containing eight $\mathrm{N}$-terminal His-residues were purified using metal-affinity chromatography. Size exclusion chromatography of the purified CCL2 showed that the protein exists as a monomer in solution (Figure S2).

Immunoblots using a CCL2-specific antiserum confirmed that CCL2 is abundant in fruiting bodies and absent from vegetative mycelium (Figure 1B). The differential expression of both CCL2 and CGLl was quantified at the transcript level by qRT-PCR (Figure 1C). The results indicate that the mRNA levels of CGL1 and CCL2 are more than 1000-fold and 60,000-fold, respectively, higher in fruiting bodies than in the vegetative mycelium.

\section{Carbohydrate-binding activity and specificity of CCL2}

Based on the binding to the plant glycoprotein HRP and a similar expression pattern as previously characterized lectins from this organism [23,24], we hypothesized that CGL2 is a lectin. Fluorescently labeled CCL2 was used to probe a glycan array offered by the Consortium of Functional Glycomics (CFG) (Figure 2 and Table S2), confirming that CGL2 is a lectin that binds specifically to carbohydrate structures containing the Fuc $\alpha 1,3 \mathrm{GlcNAc}$ motif e.g. the Lewis ${ }^{\mathrm{X}}$ antigen (Gal $\beta 1,4[\mathrm{Fu}-$ c $\alpha 1,3]$ GlcNAc; Glycan structure \#133/134 on the array). The disaccharide Fuc $\alpha 1,3 \mathrm{GlcNAc}$ alone, however, showed a very low fluorescence, suggesting that at least a trisaccharide was required for efficient binding. Glycan array analysis with purified CCL1 (Figure S3 and Table S3) yielded almost the same results as with CCL2. The binding specificity of CCL2 was further studied with several carbohydrates in vitro by NMR spectroscopy and isothermal titration calorimetry (ITC) as summarized in Table 1 . The trisaccharide Lewis ${ }^{\mathrm{X}}$ bound with a moderate $\mathrm{K}_{\mathrm{D}}$ of $456 \mu \mathrm{M}$ and the NMR spectra displayed intermediate to slow exchange behavior during the titration, whereas the binding of sialylated Lewis $^{\mathrm{X}}$, was slightly better by a factor of $\sim 3$. However, fucosylated chitobiose (GlcNAc $\beta 1,4[$ Fuc $\alpha$ 1,3] GlcNAc-spacer; Figure 3A), ab- 

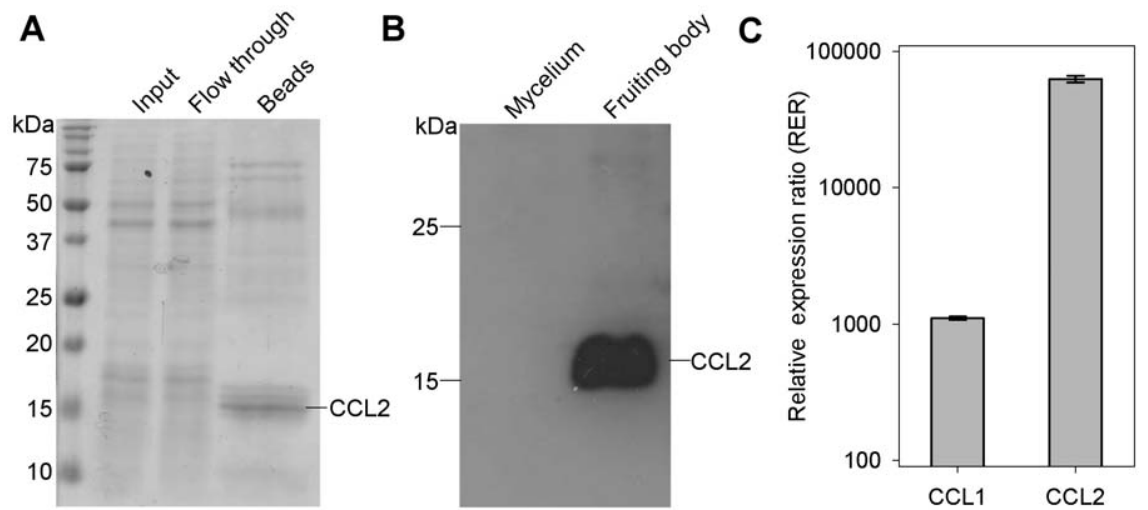

Figure 1. Isolation and differential expression of C. cinerea CCL1 and CCL2. (A) Specific binding of CCL2 to horseradish peroxidase (HRP). Coomassie-stained SDS-PAGE showing Input, Flow through and Bound (Beads) fractions of a soluble protein extract from C. cinerea fruiting bodies upon affinity-chromatography using immobilized HRP. The Bound fraction was released by boiling the HRP-sepharose beads in Lämmli sample buffer. The loaded protein amount of the Bound fraction (Beads) corresponds to two equivalents of Input and Flow through fractions. Sizes of the marker proteins are indicated. (B) Immunoblot comparing expression levels of CCL2 between vegetative mycelium and fruiting bodies of $C$. cinerea. Equal amounts of total protein were loaded in each lane. A polyclonal antiserum raised in rabbits against purified CCL2 was used for detection. (C) Comparisons of relative expression ratio (or fold up-regulation) of the genes encoding CCL1 and CCL2 by qRT-PCR in fruiting bodies relative to vegetative mycelium. Error bars represent standard deviation of the mean.

doi:10.1371/journal.ppat.1002706.g001

sent on the glycan array, had by far the highest affinity among the tested oligosaccharides with a $\mathrm{K}_{\mathrm{D}}$ of $1.4 \mu \mathrm{M}$ (Table 1 and Figure S4). Monitoring the binding by NMR spectroscopy revealed large chemical shift changes under the slow exchange regime (Figures 3B and $\mathrm{C}$ ). Binding occurs with a stoichiometry of $1: 1$ and no further changes were observed by adding an excess of ligand (1:50). The largest chemical shift deviations occurred at residues W78, N90T95, G108 and K109 (Figure 3D).

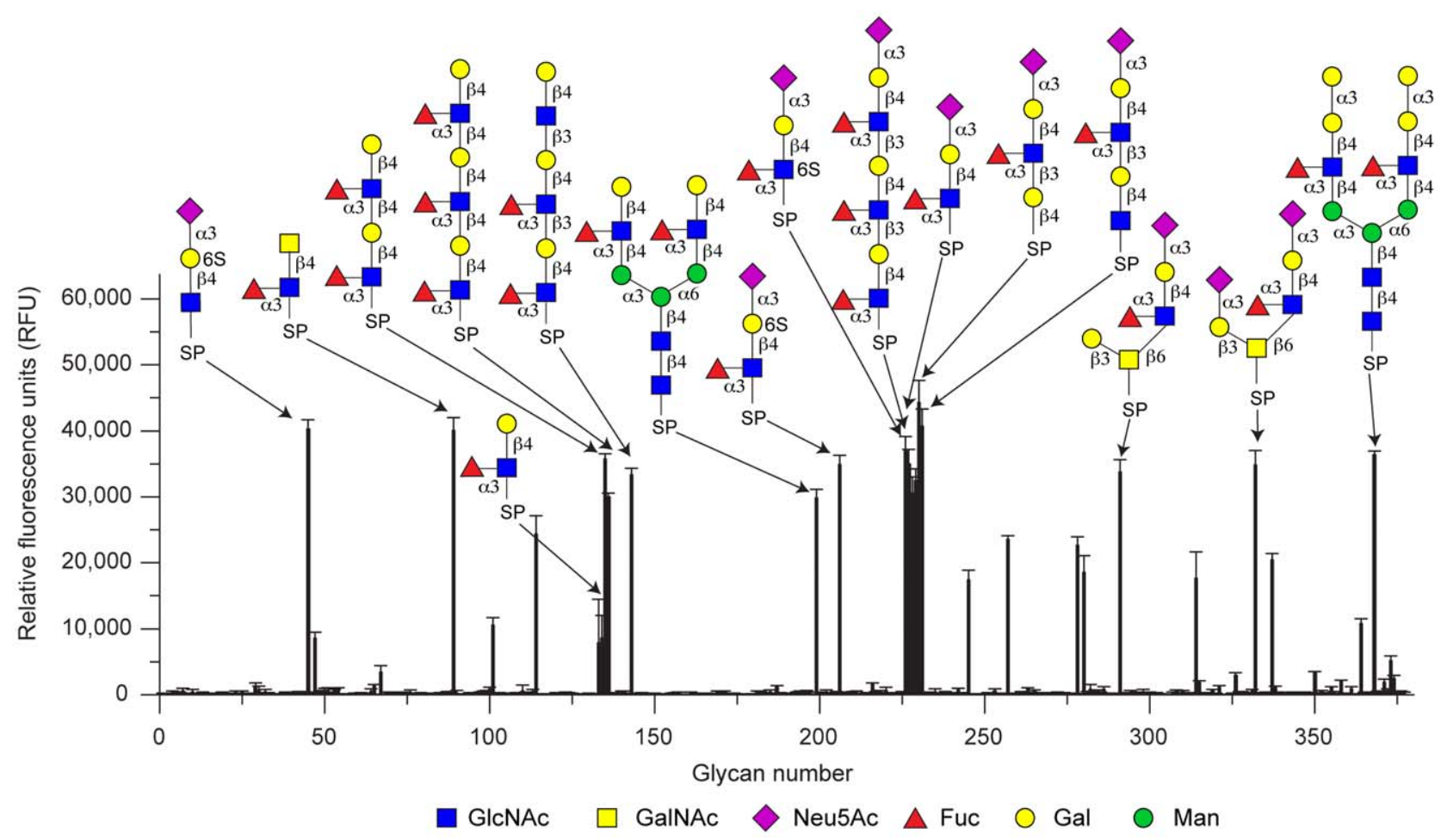

Figure 2. Carbohydrate-binding specificity of CCL2. Fluorescently labeled CCL2 was analyzed for binding to the mammalian glycan array (V3.1) of the Consortium for Functional Glycomics (CFG). Results shown are averages of triplicate measurements of fluorescence intensity at a lectin concentration of $200 \mu \mathrm{g} / \mathrm{ml}$. Error bars indicate the standard deviations of the mean. Glycan structures are depicted for those epitopes with highest relative fluorescence. The raw data and the entire list of glycans with the respective spacers can be found on the CFG homepage [http:// functionalglycomics.org/] or in Tables S2 and S3. Binding of 6'sulfo-sialyllactose (glycan \#45) is likely to be an artifact since it is also bound by fucosebinding lectin AAL [http://functionalglycomics.org/].

doi:10.1371/journal.ppat.1002706.g002 
Table 1. Binding of CCL2 wild-type to different carbohydrates and CCL2 variants to GlcNAc $\beta 1,4[\mathrm{Fuc} \alpha 1,3] \mathrm{GlcNAc} \beta 1-\mathrm{sp}$ (sp: spacer $\mathrm{O}-\left[\mathrm{CH}_{2}\right]_{5} \mathrm{COOH}$ ) measured with isothermal titration calorimetry and NMR spectroscopy at $299 \mathrm{~K}$.

\begin{tabular}{|c|c|c|c|c|c|c|c|}
\hline \multicolumn{3}{|c|}{ Carbohydrate } & $K_{D}(\mu M)$ & $\begin{array}{l}\text { Fold affinity } \\
\text { decrease }\end{array}$ & $\Delta \mathrm{H}(\mathbf{k J} / \mathbf{m o l})$ & $-\mathrm{T} \Delta \mathrm{S}(\mathrm{kJ} / \mathrm{mol})$ & NMR titration \\
\hline \multicolumn{3}{|c|}{ Fuc $\alpha 1,3 \mathrm{GICNAc-OMe}$} & $>500$ & & & & no binding \\
\hline \multicolumn{3}{|c|}{ Gal $\beta 1,4[$ Fuc $\alpha 1,3]$ GlcNAc $\beta 1-O M e$ (Le ${ }^{\mathrm{x}}$-trisaccharide) } & 456 & & & & $n d^{\mathrm{a}}$ \\
\hline \multicolumn{3}{|c|}{ Gal $\beta 1,4[$ Fuc $\alpha 1,3]$ GlcNAc $\beta 1,4$ Gal (Le ${ }^{\mathrm{x}}$-tetrasaccharide) } & $\mathrm{nd}^{\mathrm{a}}$ & & & & $\begin{array}{l}\text { large chemical shift deviations, slow } \\
\text { to intermediate exchange }\end{array}$ \\
\hline \multicolumn{3}{|c|}{ Neu5Ac $\alpha 2,3$ Gal $\beta 1,4[$ Fuc $\alpha 1,3]$ GlcNAc $\beta 1$-OMe (Sialyl Le ${ }^{x}$ ) } & 162 & & & & $\mathrm{nd}^{\mathrm{a}}$ \\
\hline \multicolumn{3}{|c|}{ GlcNAc $\beta 1,4[$ Fuc $\alpha 1,3]$ GlcNAc $\beta 1$-sp ${ }^{\text {a }}$ (Fucosylated chitobiose) } & 1.4 & & -49.8 & 16.3 & $\begin{array}{l}\text { large chemical shift deviations, slow } \\
\text { exchange }\end{array}$ \\
\hline \multicolumn{3}{|c|}{ Neu5Ac $\alpha 2,3$ Gal $\beta 1,4$ Glc (Sialyl lactose) } & $\mathrm{nd}^{\mathrm{a}}$ & & & & no binding \\
\hline Protein & KD $(\mu \mathbf{M})$ & \multicolumn{3}{|c|}{ Fold affinity decrease } & $\Delta \mathbf{H}(\mathbf{k J} / \mathrm{mol})$ & \multicolumn{2}{|c|}{$-\mathrm{T} \Delta \mathrm{S}(\mathrm{kJ} / \mathrm{mol})$} \\
\hline Wild type & 1.4 & & & & & \multicolumn{2}{|l|}{16.3} \\
\hline Y57A & 10.9 & \multicolumn{2}{|l|}{7.8} & & -44.4 & \multicolumn{2}{|l|}{15.9} \\
\hline W78A & 62.9 & \multicolumn{2}{|l|}{44.9} & & -35.6 & \multicolumn{2}{|l|}{11.7} \\
\hline L87A & 26.3 & \multicolumn{2}{|l|}{17.8} & & -43.5 & \multicolumn{2}{|l|}{17.2} \\
\hline $\mathrm{N} 90 \mathrm{~A}$ & 6.7 & \multicolumn{2}{|l|}{4.6} & & -43.1 & \multicolumn{2}{|l|}{13.4} \\
\hline N91A & $0.18^{\mathrm{b}}$ & \multicolumn{2}{|l|}{0.13} & & -57.7 & \multicolumn{2}{|l|}{18.8} \\
\hline Y92A & $>500$ & & & & & & \\
\hline V93A & 27.2 & \multicolumn{2}{|l|}{19.4} & & -39.8 & \multicolumn{2}{|l|}{13.4} \\
\hline W94A & 310 & \multicolumn{2}{|l|}{221} & & -38.1 & \multicolumn{2}{|l|}{18.0} \\
\hline K109A & 1.5 & \multicolumn{2}{|l|}{1.1} & & -47.7 & \multicolumn{2}{|l|}{14.2} \\
\hline
\end{tabular}

and: not determined.

${ }^{\mathrm{b}}$ The increased affinity of N91A might be an artifact caused by interaction of the artificial carbohydrate spacer $\mathrm{O}-\left(\mathrm{CH}_{2}\right)_{5}-\mathrm{COOH}$ with residue 91 . Whereas the spacer might sterically clash with N91, Ala in this position could form favorable van-der-Waals interactions. In the case of natural N-glycans, where the reducing GlcNAc is linked to Asn of a glycoprotein projecting away from CCL2 (upper right corner of Figure 5D), Asn is likely to be favored at this position of CCL2 due to the potential formation of $\mathrm{H}$-bonds.

doi:10.1371/journal.ppat.1002706.t001

\section{The NMR structure of CCL2 reveals a $\beta$-trefoil fold}

Since CGL2 did not show sequence similarity to any known structure we determined the 3D structure of CCL2 by NMR spectroscopy (Figure 4). CCL2 adopts a $\beta$-trefoil fold consisting of three $\beta-\beta-\beta-\beta$ repeats with a pseudo $\mathrm{C}_{3}$ symmetry. $\beta_{1}$ and $\beta_{4}$ of each repeat form together a $\beta$-barrel whereas $\beta_{2}$ and $\beta_{3}$ adopt a $\beta$ hairpin that usually harbors the carbohydrate-binding site [25]. The $\beta$-trefoil structure can be compared to a tree [26] in which the trunk is represented by the $\beta$-barrel $\left(\beta_{1}\right.$ and $\beta_{4}, \beta_{5}$ and $\beta_{8}, \beta_{9}$ and $\beta_{12}$ ), the roots are formed by the $\mathrm{N}$ - and $\mathrm{C}$-terminus together with the two loops $\beta_{4}-\beta_{5}$ and $\beta_{8}-\beta_{9}$, the upper crown is formed by the three $\beta$-hairpins $\left(\beta_{2}\right.$ and $\beta_{3}, \beta_{6}$ and $\beta_{7}, \beta_{10}$ and $\left.\beta_{11}\right)$ and the lower crown by the loops connecting the $\beta$-barrel with the $\beta$-hairpin loops. As can be seen from Figures $4 \mathrm{~B}$ and $\mathrm{D}$, the loops $\beta 6-\beta 7$ and $\beta 7-\beta 8$ in subdomain $\beta$ are shorter than in the other subdomains. In addition, subdomain $\beta$ shows a deviation from the most characteristic feature of $\beta$-trefoil proteins, the QxW motif in each subdomain [25]. Subdomain $\beta$ contains a YxW instead. A search for structurally similar proteins revealed a large number of bacterial, fungal and plant toxins displaying high structural similarity but low sequence identity (Table S4).

The 3D structure was used to visualize the largest chemical shift deviations from the titration experiment with GlcNAc $\beta 1,4[\mathrm{Fu}-$ $c \alpha 1,3]$ GlcNAc (from Figure 3D) in Figure 4C. The largest deviations occur at the interface between subdomain $\beta$ and $\gamma$, mainly on strand $\beta 8$ and its unusually short preceding loop $\beta 7-\beta 8$ ( $\beta$ subdomain) and in the $\beta 9-\beta 10$ loop ( $\gamma$ subdomain). This arrangement does not correspond to the typical binding interface of $\beta$-trefoil lectins and therefore we decided to investigate this new binding mode.

Structure of CCL2 in complex with fucosylated chitobiose

We solved the 3D structure of the complex between CCL2 and fucosylated chitobiose (GlcNAc $\beta 1,4[\mathrm{Fuc} \alpha 1,3] \mathrm{GlcNAc} \beta-\mathrm{sp})$ by NMR spectroscopy. 82 intermolecular distance restraints that are well distributed over the binding interface (Figure 5A) were derived from a 3D ${ }^{13} \mathrm{C}$ F1-edited F3-filtered HSQC-NOESY [27] spectrum (Figure S5). A precise structural ensemble of the complex was obtained (Figure 5B and Table 2). The carbohydrate is bound at the interface of the subdomains $\beta$ and $\gamma$ in the lower crown (Figure $5 \mathrm{C}$ ), in particular between the $\beta$-strands $\beta_{6}$ and $\beta_{8}$ and the linker $\beta_{7}-\beta_{8}$ of the $\beta$ subdomain and the loop between $\beta_{9}-\beta_{10}$ of the $\gamma$ subdomain. Compared to the canonical binding sites (Figure 5G and Figure S6) this is a very unusual binding location for ricin $\mathrm{B}$ type lectins.

The well-defined trisaccharide is oriented such that GlcNAc2 (see Figure 3A for nomenclature of the individual sugars in the trisaccharide) stacks on top of Fuc2' thereby locking the conformational freedom of the glycan resulting in a narrow clustering of the glycosidic angles (Figure S7). The hydrophobic Bface of Fuc2' is oriented towards the protein (bottom) and the hydrophobic B-face of GlcNAc2 towards the solution (top). In this orientation GlcNAcl is tilted horizontally such that its B-face is located on the back contacting the protein. Contacts to all three sugar units are mediated by a large number of potential $\mathrm{H}$-bonds and hydrophobic interactions (Figures 5D-F and Table 3). 
A
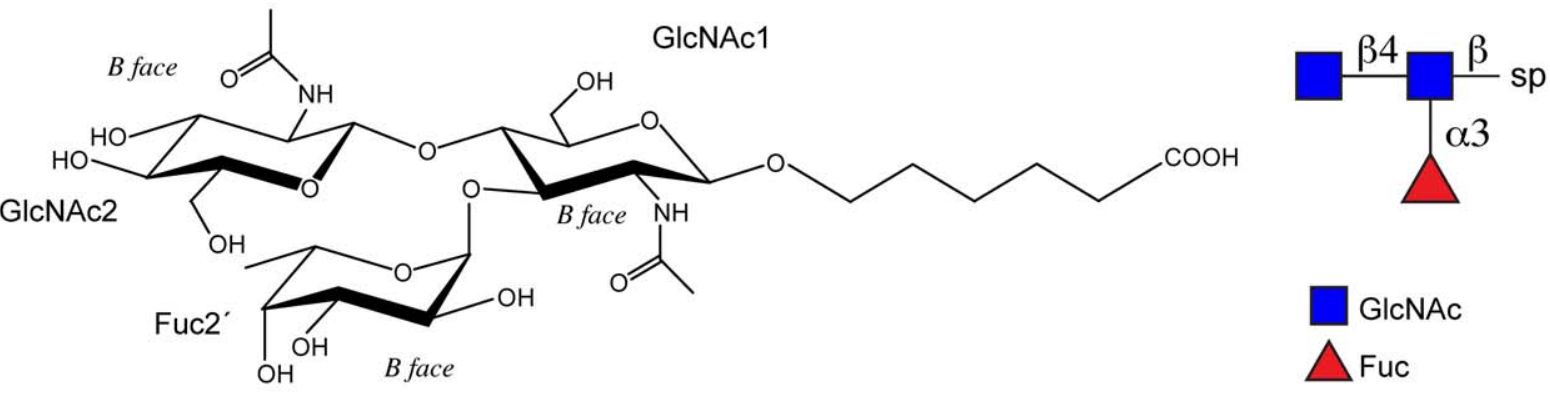

B

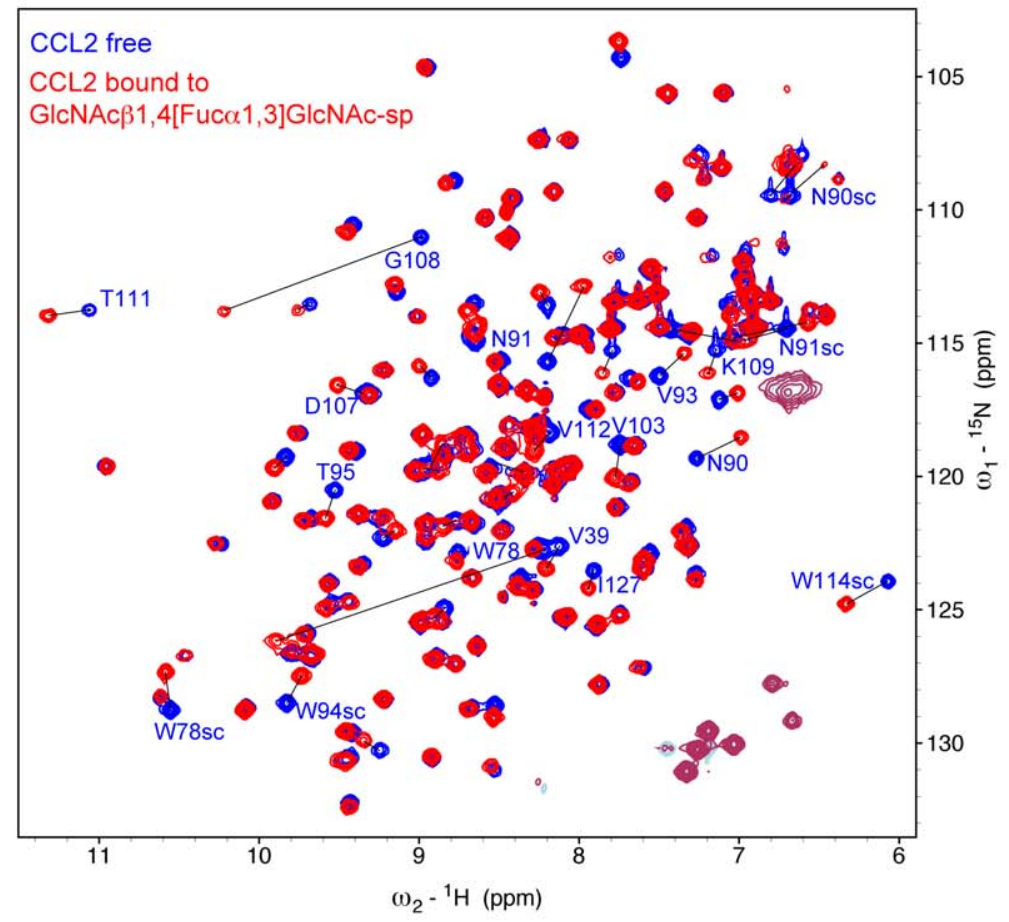

\section{C}

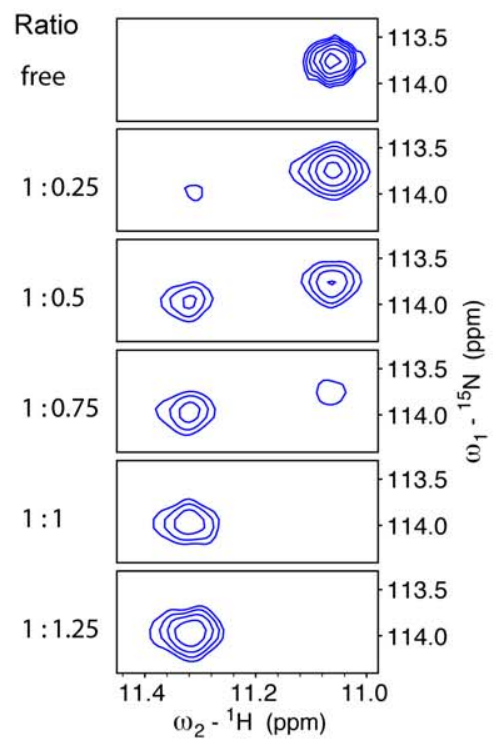

D

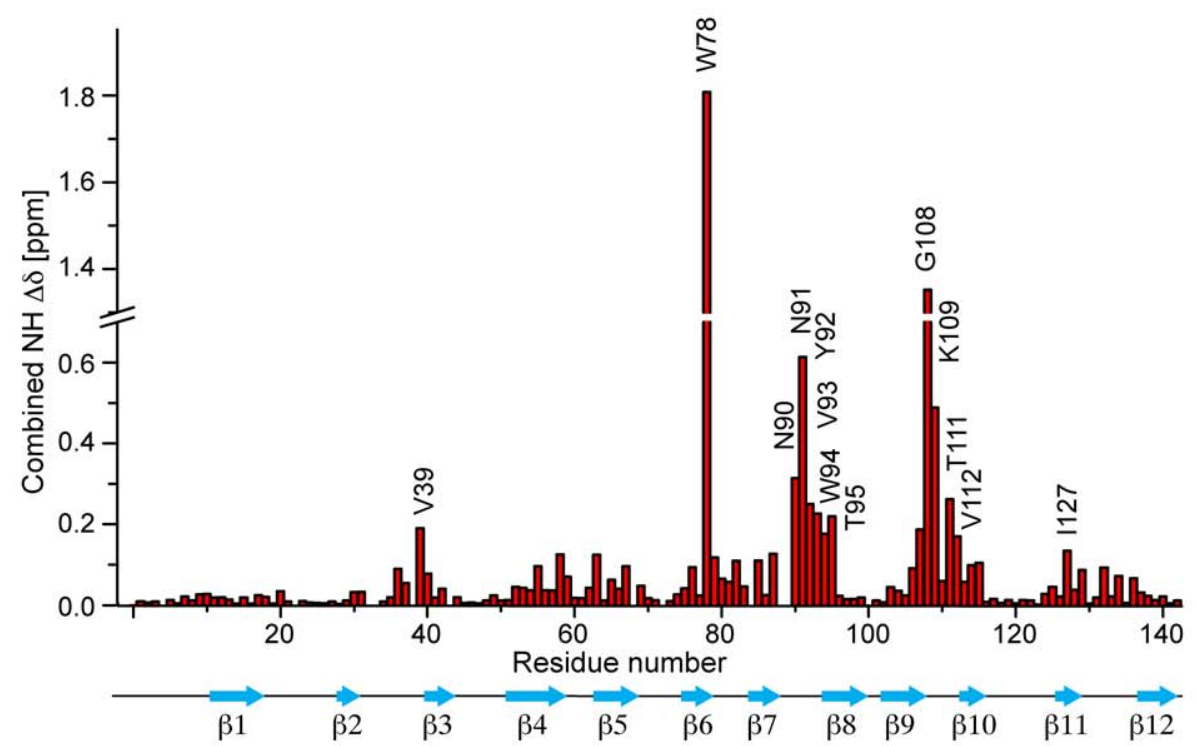

Figure 3. Refining the specificity of the CCL2 lectin. (A) The chemical and schematic structure of the fucosylated chitobiose (GlcNAc $\beta 1,4[F u c \alpha 1,3]$ GlcNAc-spacer) that was used as ligand for binding studies and structure determination. Indicated is also the $B$ face that is defined as the face on which the carbons are numbered in an anticlockwise order [69]. (B) Chemical shift deviations upon complex formation at a protein concentration of $0.4 \mathrm{mM}$ at pH 5.7. Overlay of ${ }^{15} \mathrm{~N}-\mathrm{HSQC}$ spectra of free CCL2 (blue) and CCL2 bound to one equivalent of fucosylated chitobiose (red). (C) Titration of the amide signal of T111 in CCL2 with fucosylated chitobiose using ${ }^{15} \mathrm{~N}-\mathrm{HSQC}$ spectra. The protein:ligand ratio is displayed on the left. (D) Plot of the chemical shift differences between free and bound CCL2 $\left(\delta=\left[\delta_{\mathrm{HN}}{ }^{2}+\left(\delta_{\mathrm{N}} / R_{\mathrm{scale}}\right)^{2}\right]^{1 / 2}, \mathrm{R}_{\mathrm{scale}}=5\right)$. doi:10.1371/journal.ppat.1002706.g003 
A

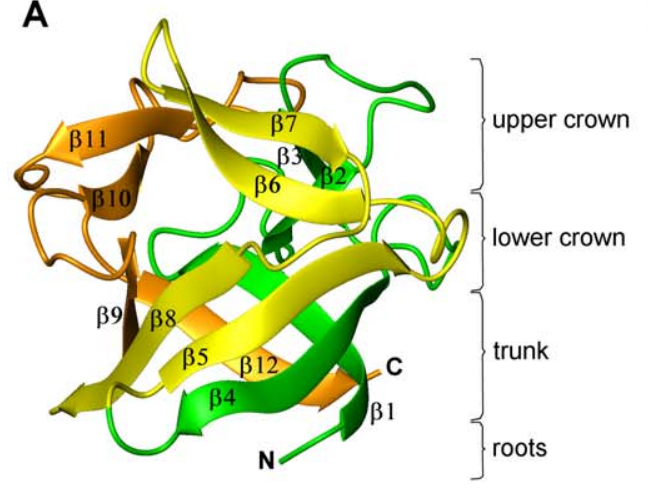

B

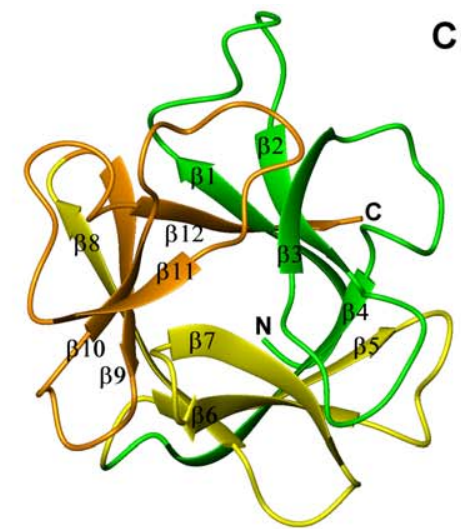

C

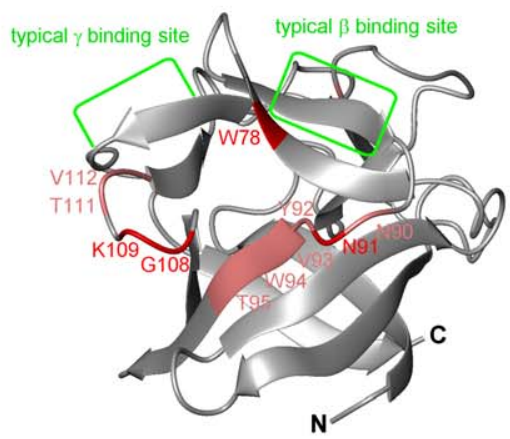

D

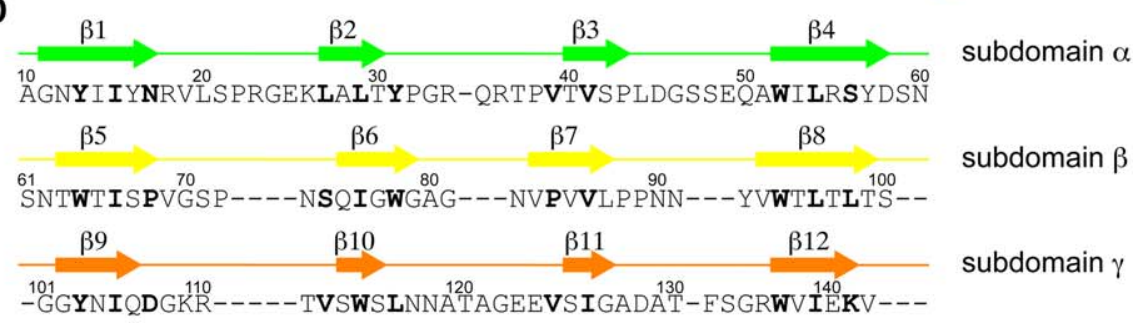

Figure 4. Solution structure of the CCL2 lectin in the absence of a ligand determined by NMR spectroscopy. The side (A) and top (B) view of the most representative structure out of 20 structures is shown. The three pseudo symmetric sections of the $\beta$-trefoil fold corresponding to residues S9-N60, S61-S100 and G101-V142 are colored green, yellow and orange, respectively. Characteristic regions are labeled according to Renko et al. for better orientation [26]. (C) Chemical shift deviations mapped on the structure of CCL2 in the same orientation as in A. Chemical shifts of residues in red experience a combined $\mathrm{NH}$ chemical shift deviation $>0.4 \mathrm{ppm}$, for residues in pink $>0.15 \mathrm{ppm}$. (D) Secondary structure and subdomain borders displayed on the protein sequence. The same color code as in A and B is used. Bold residues are forming the hydrophobic core of the protein.

doi:10.1371/journal.ppat.1002706.g004

The specific recognition of each sugar unit can be described as follows: Fuc2' approaches the edge of $\beta$-strand $\beta 8$ and the tip of loop $\beta 9-\beta 10$ with its b-face and bridges subdomain $\beta$ and $\gamma$ in this way (Figures 5C-F). In this orientation $\mathrm{O} 4$ and $\mathrm{O} 5$ face down and are specifically recognized by H-bonds to the main chain (V93 HN and O) of the unusually short loop between strands $\beta 7$ and $\beta 8$ (Figure $5 \mathrm{E}$ ). The equatorial hydroxyl groups of $\mathrm{O} 3$ and $\mathrm{O} 2$ form $\mathrm{H}$-bonds to G108 HN (second largest chemical shift deviation, Figure 3D) and Lys $109 \mathrm{NH}_{3}{ }^{+}$. In addition the hydrophobic methyl group and the axial $\mathrm{H} 2$, both facing downwards, form hydrophobic contacts with Trp94/Trp95 and Val93, respectively. The methyl group is located above the ring of W94 enabling favorable Me- $\pi$ interactions that are supported by an upfield shift of the $\mathrm{H} 6$ resonance $(-0.18 \mathrm{ppm}$; Table S5). In total all characteristic groups of Fuc2' are specifically recognized by $4 \mathrm{H}$-bonds, hydrophobic and $\pi$ interactions. Both the location of Fuc2' at the subdomain interface and the recognition by three $\mathrm{H}$-bonds to the main-chain are unprecedented in all ricin $\mathrm{B}$ type lectin complex structures. GlcNAcl is specifically recognized at its equatorial acetamido group by a H-bond of its $\mathrm{HN}$ to Asn91 O, and at $\mathrm{O} 6$ by an H-bond to the side chain of Asn90. The acetamido group forms hydrophobic interactions to Val93 and Me- $\pi$ interactions with Tyr57 which is supported by an upfield shift of the methyl ${ }^{1} \mathrm{H}$ resonance $(-0.24 \mathrm{ppm})$. Its hydrophobic b-face packs to the Tyr92 side chain. Only a GlcNAc would be recognized at this position since the equatorial orientation of the acetamido and the $\mathrm{CH}_{2} \mathrm{OH}$ group are necessary for their recognition by $\mathrm{H}$-bonds and the equatorial positioning of $\mathrm{O} 3$ and $\mathrm{O} 4$ is required for the stacking between Fuc2' and GlcNAc2. GlcNAc2 is mainly recognized via its acetamido group by an H-bond to Trp78 HN (supported by the largest $\mathrm{HN}$ chemical shift deviation, Figure 3D), hydrophobic interactions of the methyl with Leu87 and a stacking of the entire acetamido group to the ring of Tyr92 (Figure 5D). Me- $\pi$ interactions to $\mathrm{Y} 92$ are supported by an upfield shift $(-0.24 \mathrm{ppm})$. GlcNAc2 that stacks on top of Fuc2' is slightly laterally shifted exposing the hydrophobic $\mathrm{H} 4$ facing downwards. $\mathrm{H} 4$ is located on top of the Trp94 ring and favorable $\mathrm{H}-\pi$ interactions are supported by an upfield shift of its resonance ( $-0.39 \mathrm{ppm}$; Table S5). Two additional potential $\mathrm{H}$-bonds are observed in some structures of the ensemble: between the carbonyl of W78 and O3 of GlcNAc2 and between $\mathrm{K} 109 \mathrm{NH}_{3}{ }^{+}$and GlcNAc2 O6. In summary, GlcNAc1 and Fuc2' are specifically recognized by interactions to almost all of their functional groups whereas the recognition of GlcNAc2 is more relaxed. It is mainly recognized at its equatorial acetamido group attached to $\mathrm{C} 2$. This residue must be able to stack to Fuc2' in order to properly position the acetamido group; both GlcNAc and GalNAc fulfill this requirement and will be recognized in this position. Accordingly, CCL2 binds to fucosylated LacdiNAc (GalNAc $\beta 1,4[$ Fuc $\alpha 1,3]$ GlcNAc; Glycan structure \#89) on the array.

The large number of $\mathrm{H}$-bonds to the main chain is remarkable. The unusually short $\beta 7-\beta 8$ loop contributes three and the $\beta 9-\beta 10$ loop one such H-bonds. Since the protein main chain does not change upon binding, part of the recognition pattern on the protein is preformed. However, the lengths and conformations of these loops are a special feature of CCL2 homologues as illustrated on a structure-based alignment (Figure 6) and are not conserved in the $\beta$-trefoil fold. Note also that the short $\beta 7-\beta 8$ loop lacks the typical $3_{10}$ helix segment as seen for example in the structurally most closely related R-type lectin MOA (Figure 5G) which would clash with the carbohydrate. 
A

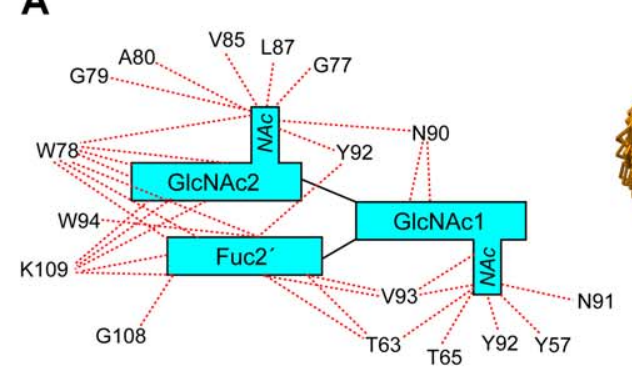

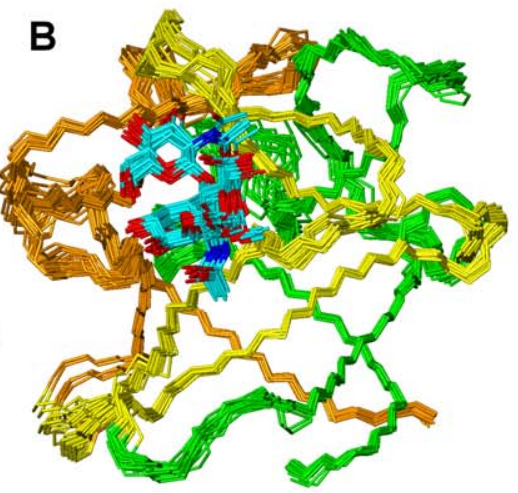
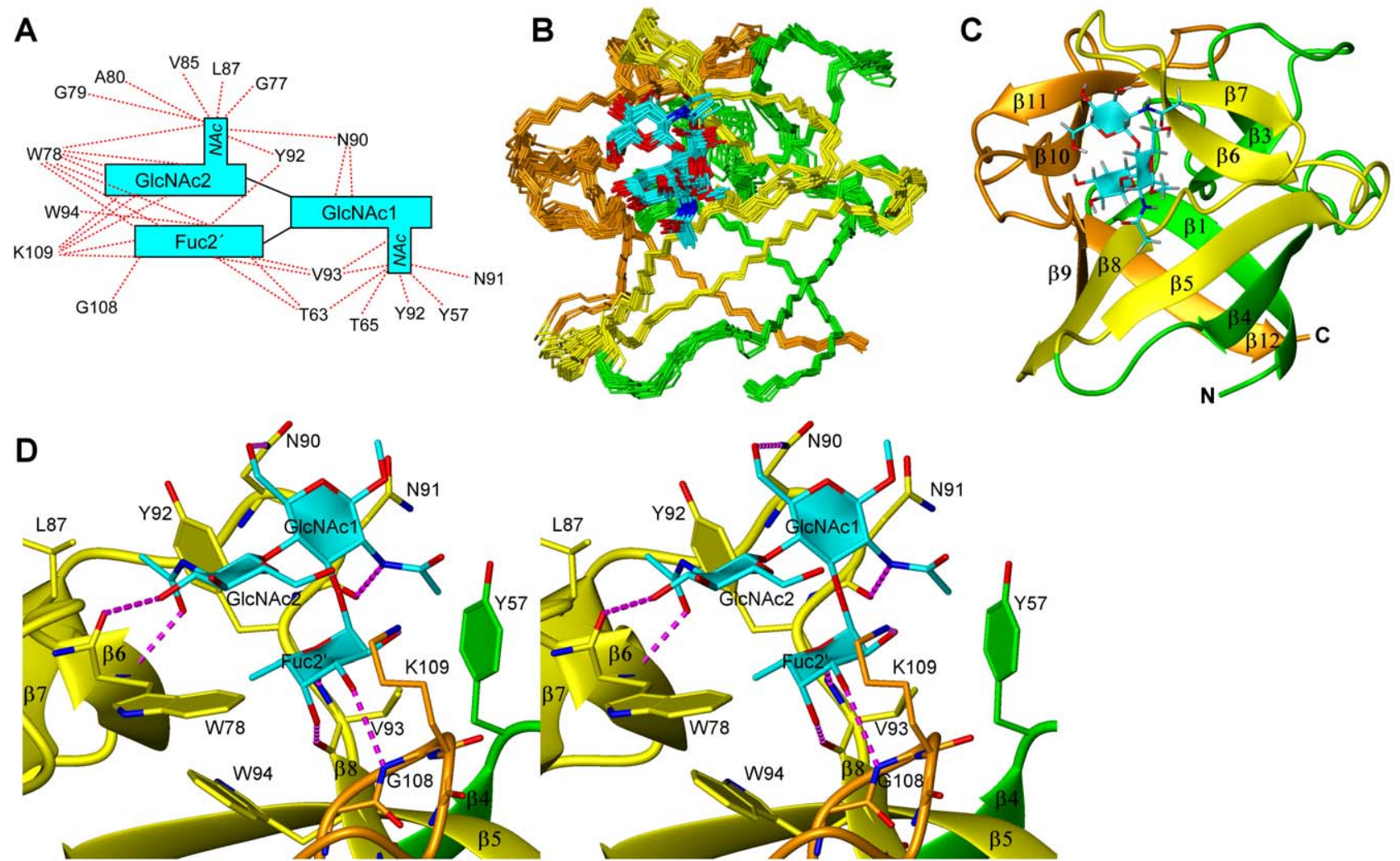

E

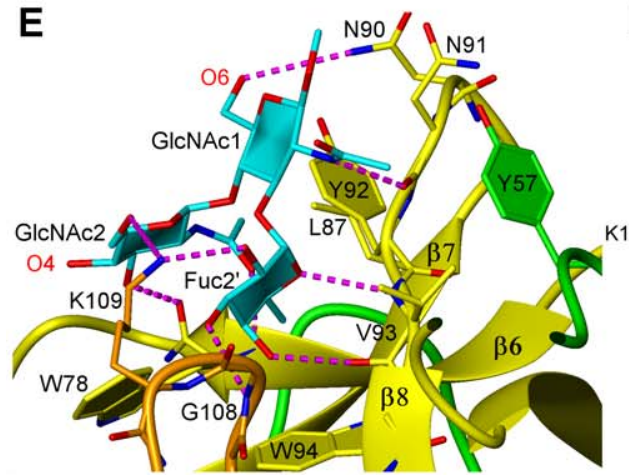

$\mathbf{F}$

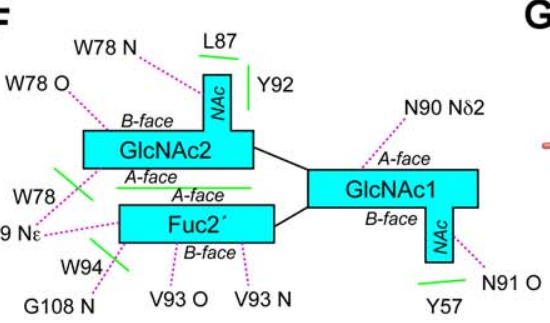

G

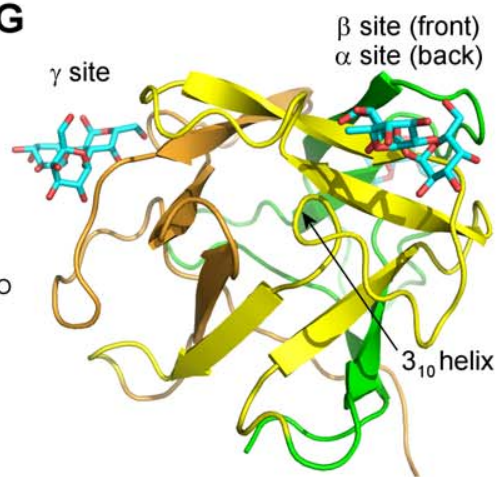

Figure 5. NMR solution structure of the CCL2 lectin in complex with fucosylated chitobiose (GIcNAcB1,4[Fuca1,3]GIcNAc). (A) Intermolecular NOEs observed in a 3D ${ }^{13} \mathrm{C}$ F1-edited F3-filtered HSQC-NOESY spectrum in a schematic presentation. (B) Structural ensemble of 20 structures of the protein backbone and the carbohydrate in cyan. The subunits $\alpha, \beta$ and $\gamma$ are colored green, yellow and orange, respectively. The orientation is identical to Figure 4. (C) Ribbon presentation of the most representative structure. (D) Stereo view of the carbohydrate recognition site. Potential intermolecular hydrogen bonds are shown with dashed magenta lines. (E) Details of the interaction site illustrating how the trisaccharide is recognized by hydrogen bonds. (F) Summary of the interactions between the trisaccharide and CCL2. Potential H-bonds are indicated as dotted lines in magenta and hydrophobic interactions by green lines. (G) Crystal structure of the $\beta$-trefoil domain of the fungal lectin MOA in complex with the trisaccharide Gal $\alpha 1,3[\mathrm{Fuc} \alpha 1,2] \mathrm{Gal}$ [19] showing all three occupied canonical binding sites (pdb:3EF2). For better comparison, the same orientation and colors as in panel $\mathrm{B}$ and $\mathrm{C}$ were used.

doi:10.1371/journal.ppat.1002706.g005

\section{Comparison of binding affinity and thermodynamics with other lectins}

The interaction of CCL2 with GlcNAc $\beta 1,4[\mathrm{Fuc} \alpha 1,3] \mathrm{GlcNAc}$ is governed by a large $\Delta \mathrm{H}$ gain of $-50 \mathrm{~kJ} \mathrm{~mol}^{-1}$ at the expense of $16 \mathrm{~kJ} \mathrm{~mol}^{-1}$ for $-\mathrm{T} \Delta \mathrm{S}$ (Figure $7 \mathrm{~A}$ ). The thermodynamicbinding parameters are comparable to those of other high affinity lectins in Figure 7B (Table S6). In contrast to typical lectin interactions with medium affinity CCL2 uses an unusually large number of $\mathrm{H}$-bonds (5-7 to backbone, 5 to side chain) and hydrophobic contacts (Trp78, Tyr92 and Trp94) for recognition of its target. A comparable number and kind of contacts is only found for few high affinity lectin interactions with a comparable $\mathrm{K}_{\mathrm{D}} \sim 1 \mu \mathrm{M}$. Interestingly, the calreticulin interaction with Glc $\alpha 1,3 \mathrm{Man} \alpha 1,2 \mathrm{Man} \alpha 1,2 \mathrm{Man}$ with a $\mathrm{K}_{\mathrm{D}}$ of $0.77 \mu \mathrm{M}$ is governed by almost identical thermodynamic values [28], whereas the structurally closely related R-type lectin PSL [20] that binds to 6'sialyl lactose with a $\mathrm{K}_{\mathrm{D}}$ of $1.3 \mu \mathrm{M}$ [29] displays a moderately favored enthalpy but almost no entropic penalty. Both lectins use a similar number of direct $\mathrm{H}$-bonds for their target recognition as CCL2 does: 10 (2 to backbone, 8 to side chain) and 9 (4 to the backbone, 5 to side chains), respectively, and a comparable amount of hydrophobic interactions. 
Table 2. NMR structure determination statistics of CCL2 in the free form and in complex with the fucosylated chitobiose (GlcNAc $\beta 1,4[\mathrm{Fuc} \alpha 1,3] \mathrm{GlcNAc-spacer}$, the spacer $\left[\mathrm{CH}_{2}\right]_{5} \mathrm{COOH}$ was truncated in the structure calculations to a methyl group.).

\begin{tabular}{|c|c|c|c|}
\hline & \multirow[b]{2}{*}{ CCL2 free } & \multicolumn{2}{|c|}{ CCL2-carbohydrate complex } \\
\hline & & CCL2 & carbohydrate \\
\hline \multicolumn{4}{|l|}{ NMR distance and dihedral constraints } \\
\hline \multicolumn{4}{|l|}{ Distance restraints } \\
\hline Total NOE & 2514 & 2054 & 42 \\
\hline Intra-residue & 482 & 446 & 23 \\
\hline Inter-residue & 2032 & 1608 & 19 \\
\hline Sequential $(|i-j|=1)$ & 593 & 489 & 9 \\
\hline Nonsequential $(|i-j|>1)$ & 1439 & 1119 & 10 \\
\hline Hydrogen bonds & 46 & 49 & - \\
\hline Protein-carbohydrate intermolecular & & 82 & \\
\hline Total dihedral angle restraints & 186 & 178 & \\
\hline \multicolumn{4}{|l|}{ Protein } \\
\hline$\phi$ & $91^{*}$ & $85^{*}$ & \\
\hline$\psi$ & 95 & 91 & \\
\hline \multicolumn{4}{|l|}{ Carbohydrate } \\
\hline HN-CO peptide bonds of acetamido & & & 2 \\
\hline \multicolumn{4}{|l|}{ Structure statistics } \\
\hline \multicolumn{4}{|l|}{ Violations (mean and s.d.) } \\
\hline Number of distance constraint violations $>0.2 \AA$ & $0.42 \pm 0.64$ & $0.45 \pm 0.61$ & \\
\hline Number of dihedral angle violations $>5^{\circ}$ & $0.05 \pm 0.22$ & $4.75 \pm 1.48$ & \\
\hline Max. dihedral angle violation $\left({ }^{\circ}\right)$ & $2.6 \pm 3.4$ & $17.4 \pm 8.5$ & \\
\hline Max. distance constraint violation $(\AA ̊)$ & $0.24 \pm 0.07$ & $0.20 \pm 0.04$ & \\
\hline \multicolumn{4}{|l|}{ Deviations from idealized geometry } \\
\hline Bond lengths $(\AA)$ & 0.010 & 0.010 & \\
\hline Bond angles $\left({ }^{\circ}\right)$ & 2.29 & 2.38 & \\
\hline \multicolumn{4}{|l|}{ Average pairwise r.m.s. deviation ${ }^{* *}(\AA)$} \\
\hline \multicolumn{4}{|l|}{ Protein (residues G22-V153) } \\
\hline Heavy & $0.98 \pm 0.14$ & $1.18 \pm 0.15$ & \\
\hline Backbone & $0.45 \pm 0.10$ & $0.71 \pm 0.13$ & \\
\hline \multicolumn{4}{|l|}{ carbohydrate } \\
\hline All glycan heavy & & & $0.47 \pm 0.21$ \\
\hline \multicolumn{4}{|l|}{ Complex } \\
\hline Protein and carbohydrate heavy & & $1.17 \pm 0.15$ & \\
\hline
\end{tabular}

\section{Biotoxicity of CCL2 against invertebrates and its dependence on specific carbohydrate binding}

We tested the toxicity of CCL2 against four model organisms: the nematode Caenorhabditis elegans, the insects Aedes aegypti and Drosophila melanogaster, and the amoeba Acanthamoeba castellanii. The biotoxicity assays were performed either by feeding the test organisms with $E$. coli expressing the recombinant lectin as described previously [30], or by adding the purified lectin to the food source of the organisms.

These experiments showed a toxicity of CCL2 for C. elegans and D. melanogaster (Figure 8) but not for A. aegypti or A. castellanii (Figure S8). In the case of C. elegans, feeding on CCL2-expressing E. coli stopped the development of all wildtype (N2) L1 larvae in the assay (Figure 8B). This toxicity was dose-dependent and the presence of
$30 \%$ of CCL2-expressing E. coli among the fed bacteria was sufficient to reduce the development of more than $95 \%$ of the L1 larvae (Figure S9). In the case of D. melanogaster, CCL2 caused a significant delay in development of both pupae and flies by 4 - and 10 -fold, respectively, relative to the control (Figure 8D). The toxicity of CCL1 towards C. elegans (Figure S10) was found to be similar to that of CCL2.

The observed toxicity was likely to be mediated by binding of CCL2 to the N-glycan cores of glycoproteins in the susceptible organisms since $\alpha 1,3$-fucosylation of $\mathrm{N}$-glycan cores was demonstrated both for C. elegans and D. melanogaster and caused crossreactivity of anti-HRP antisera with these organisms [31]. Therefore, C. elegans mutants impaired in either fucose biosynthesis (bre-1) or a variety of fucosyltransferases were tested for their 
Table 3. Potential intermolecular protein-carbohydrate hydrogen bonds based on the orientations and positions of the carbohydrate in the complex structure.

\begin{tabular}{|c|c|c|}
\hline Hydrogen bonds & Occurence in ensemble $<3.2 \AA$ & Supporting chemical shift \\
\hline W78 N - GlcNAc2 O7 & $15 / 20$ & largest $\mathrm{NH}$ chemical shift change (Figures 3B and D) \\
\hline W78 O - GlcNAC2 O3 & $1 / 20$ & small $C^{\prime}$ chemical shift change $\delta\left(C^{\prime}\right)_{\text {free }}=175.9 \mathrm{ppm} ; \delta\left(C^{\prime}\right)_{\text {bound }}=176.1 \mathrm{ppm}$ \\
\hline N90 ND2 - GlcNAc1 O6 & $6 / 20$ & large $\mathrm{NH}$ chemical shift change (Figure $3 \mathrm{~B}$ ) \\
\hline N91 O - GlcNAc1 N2 & $19 / 20$ & moderate $C^{\prime}$ chemical shift change $\delta\left(C^{\prime}\right)_{\text {free }}=173.3 \mathrm{ppm} ; \delta\left(C^{\prime}\right)_{\text {bound }}=173.8 \mathrm{ppm}$ \\
\hline V93 N - Fuc2' O5 & $5 / 20$ & moderate NH chemical shift change (Figures 3B and D) \\
\hline V93 O - Fuc2' O4 & $20 / 20$ & largest $C^{\prime}$ chemical shift change $\delta\left(C^{\prime}\right)_{\text {free }}=174.4 \mathrm{ppm} ; \delta\left(C^{\prime}\right)_{\text {bound }}=172.2 \mathrm{ppm}$ \\
\hline G108 N - Fuc2' O3 & $12 / 20$ & second largest NH chemical shift change (Figures $3 \mathrm{~B}$ and $\mathrm{D}$ ) \\
\hline G108 N - Fuc2' 04 & $17 / 20$ & second largest NH chemical shift change (Figures 3B and D) \\
\hline K109 NE - Fuc2' O2 & $4 / 20$ & \\
\hline K109 NE - Fuc2' O3 & $3 / 20$ & \\
\hline K109 NE - GlcNAc2 O4 & $4 / 20$ & \\
\hline K109 NE - GlcNAc2 O6 & $10 / 20$ & \\
\hline
\end{tabular}

Note that no intermolecular hydrogen bond constraints were used during the calculations.

doi:10.1371/journal.ppat.1002706.t003

susceptibility to CGL2-mediated toxicity (see scheme in Figures 8A and B; Figure S11). In agreement with our predictions, the bre1(ye4) mutant that is unable to synthesize GDP-fucose was completely resistant to CCL2 intoxication. In addition, the fut1(ok892) mutant lacking Fuc $\alpha 1,3$ at the proximal GlcNAc of the chitobiose core [32] was partially resistant, as most of the worms survived and developed, but just half of the larvae reached L4 stage after 48 hours. On the other hand, a deletion in the fut- 6 gene, which results in loss of tetrafucosylated N-glycans in $C$. elegans, as does a deletion in the fut-1 gene [32], was as sensitive as N2 (wildtype) to CCL2. In order to further explore these results, a fut-6(ok475)fut-1(ok892) double mutant was constructed and found to be completely resistant to CGL2 (Figure 8B). As nematodes are able to $\alpha 1,3$-fucosylate both GlcNAc residues of the core region of some N-glycans [33] and both fut- 1 and fut- 6 are required for the full fucosylation of this core region (see scheme in Figure 8A; Yan, Paschinger and Wilson, personal communication), our results suggest that the $\alpha 1,3$-fucosylated chitobiose core of $\mathrm{N}$-glycans is the ligand of CCL2 in C. elegans. The partial resistance of the fut-1 mutant can be explained by binding of CCL1/2 to N-glycan cores carrying a single fucose on the distal GlcNAc (Man $\beta 1,4[\mathrm{Fu}-$ $\mathrm{c} \alpha 1,3] \mathrm{GlcNAc})$. We hypothesize that this is a less favorable ligand due to the lack of an acetamido group on the mannose.

To study the phenotype of CCL2-mediated intoxication and to follow the fate of the toxic lectin in the worms, different C. elegans strains were fed with $E$. coli cells producing an N-terminal fusion of CGL2 to the red fluorescent dTomato protein [34]. As can be observed in Figure 8C, a strong fluorescence was observed in the upper intestine of the completely susceptible worms $\mathrm{N} 2$ and fut$6(0 k 475)$ as a result of CCL2-binding to the intestinal epithelium. This fluorescence was accompanied by an evident damage of intestinal cells which resulted in a massive expansion of the intestinal lumen. In agreement with the effects on larval development (Figure 8B), the fut-6(ok475)fut-1(ok892) double mutant that is resistant to CCL2-mediated intoxication, showed neither red fluorescence nor cell damage or expansion of the intestinal lumen. These results suggest that, in the absence of binding to the intestinal epithelium, the ingested lectin is completely cleared from the lumen after 1 hour. Accordingly, an intermediate phenotype, with some staining and cell damage, mostly in the upper part of the intestinal epithelium, was observed in the partially resistant fut-1(ok892) mutant.

\section{Effect of point mutations on carbohydrate-binding affinity and toxicity}

We evaluated the contribution of individual amino acid side chains on the carbohydrate-binding affinity by introducing several point mutations at the binding interface followed by ITC measurements. All variants expressed well (except N91A) and folded properly as judged from ${ }^{15} \mathrm{~N}-\mathrm{HSQC}$ spectra (Figure S12). Significant decreases in affinity were observed for all mutants except N91A (Table 1 and Figure S13). The Y92A mutation decreased the affinity beyond the detection limit. The second largest affinity decreases are observed for W94A and W78A, indicating that the aromatic side chains provide the largest contribution to carbohydrate-binding affinity. A significant decrease in affinity was also observed for Y57A, L87A, N90A and V93A point mutants (4- to 17 -fold).

GCL2 variants were also tested in vivo for toxicity towards $C$. elegans. Remarkably, those mutants that retained carbohydrate binding with high affinity $\left(\mathrm{K}_{\mathrm{D}}<30 \mu \mathrm{M}\right)$ in vitro were as toxic as wild type CCL2. Mutants with lower in vitro affinity, however, showed a decreased toxicity towards C. elegans (Figure 8E). In summary, these results confirm the carbohydrate-coordinating residues of CCL2 that were identified by NMR spectroscopy and suggest that high carbohydrate-binding affinity of the lectin is required for toxicity.

\section{Discussion}

Our results strongly suggest that the newly identified lectins play a role in fungal defence. The lack of motility and the resulting inability of multicellular fungi and plants to escape from predators and parasites has led to the development of very similar defence strategies. In the absence of adaptive immune mechanisms and circulating immune cells, both types of organisms solely rely on innate defence. Whereas plant defence has already been intensively studied [9,35-37], fungal defence has only recently been explored. It appears that, similar to plants, in addition to small molecules [38], proteins play a key role in the defence of 


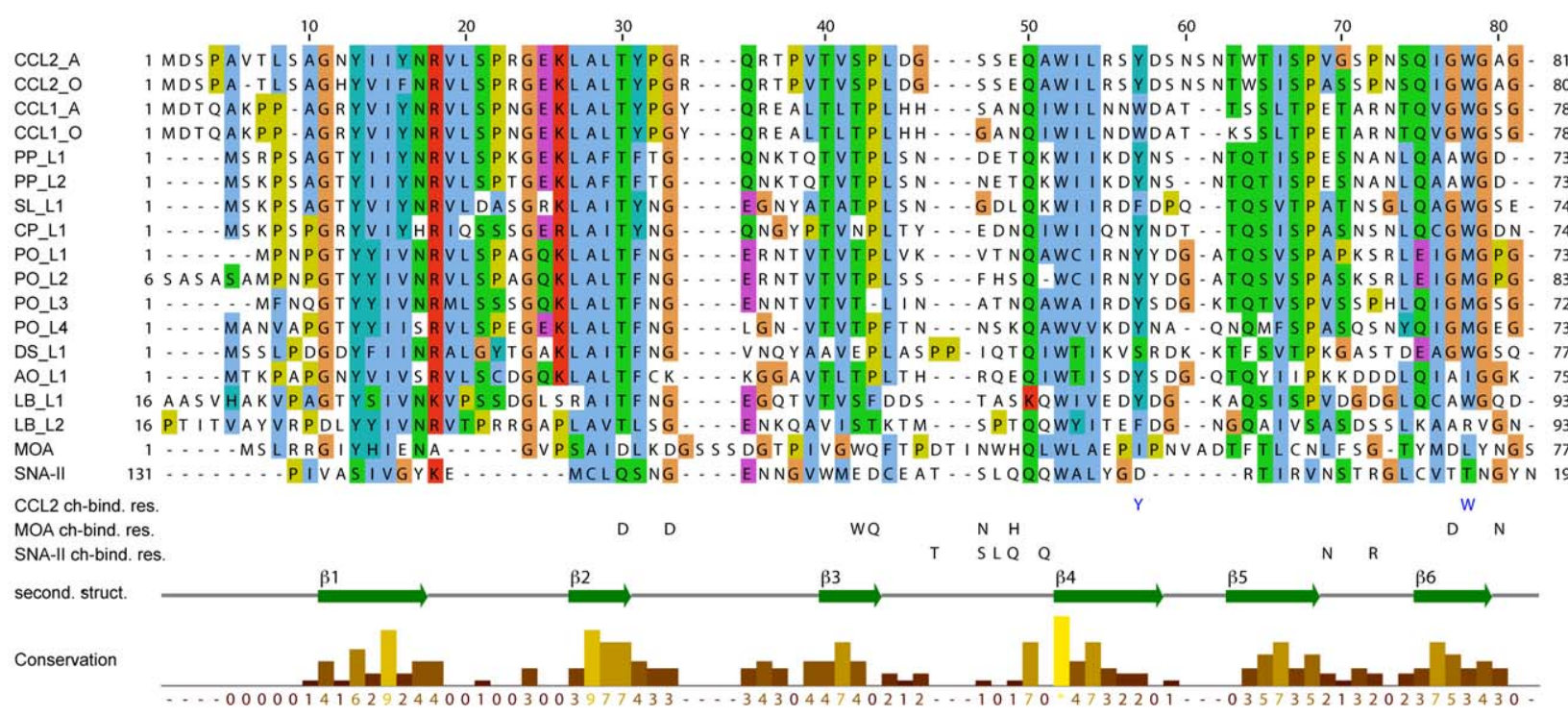

\section{0}

100

110

120

130

140

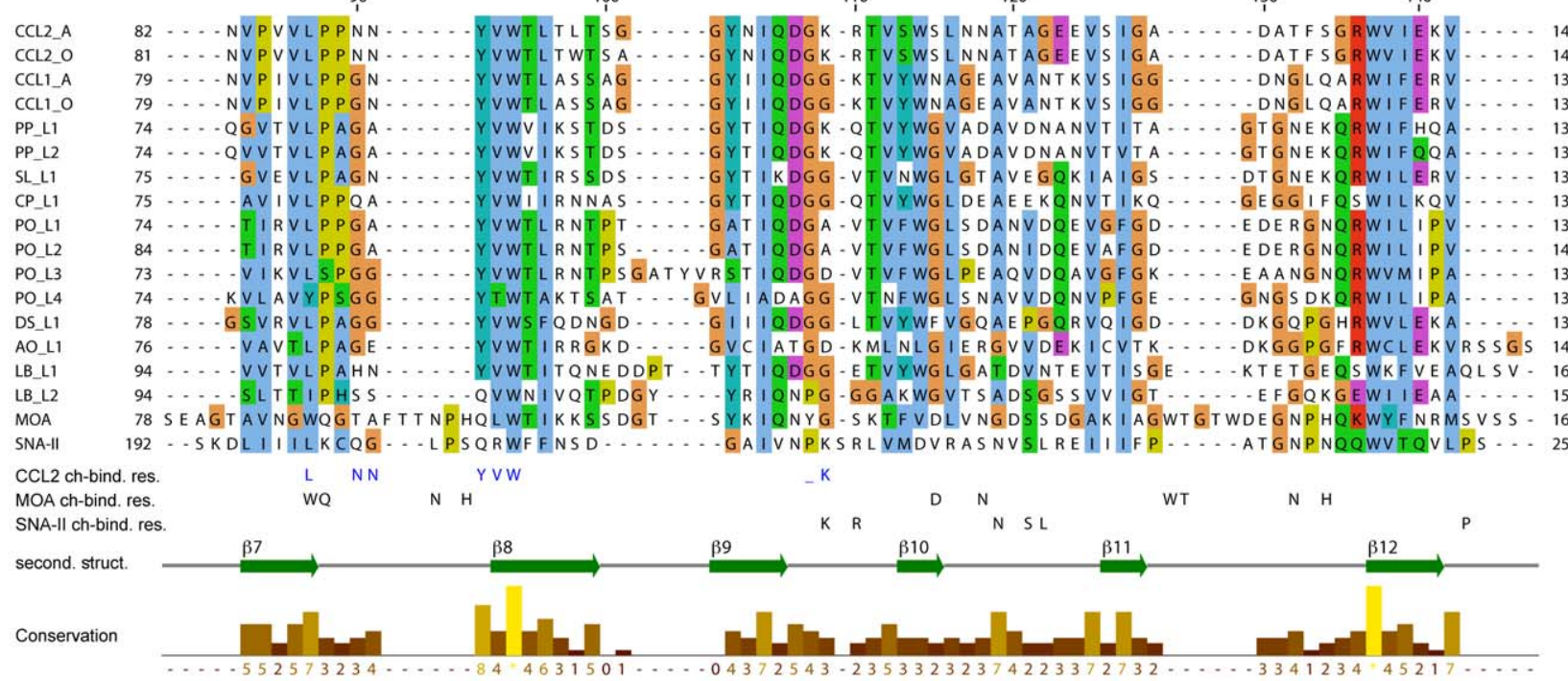

Figure 6. Sequence conservation among CCL2-like proteins and comparison to two typical representatives of fungi and plants. Sequence alignment of several fungal and plant R-type lectins. CCL2_A: CCL2 of C. cinerea strain AmutBmut; CCL2_O: CCL2 of C. cinerea strain Okayama7; CCL1_A: CCL1 of C. cinerea strain AmutBmut; CCL1_O: CCL1 of C. cinerea strain Okayama7; PP_L1: Postia placenta lectin 1 (Pospl1_130016); PP_L2: Postia placenta lectin 2 (Pospl1_121916); SL_L1: Serpula lacrymans lectin 1 (SerlaS7_144703); CP_L1: Coniophora puteana lectin 1 (Conpu1_119225); PO_L1: Pleurotus ostreatus lectin 1 (PleosPC9_89828); PO_L2: Pleurotus ostreatus lectin 2 (PleosPC15_1043947); PO_L3: Pleurotus ostreatus lectin 3 (PleosPC9_64199); PO_L4: Pleurotus ostreatus lectin 4 (PleosPC15_1065820); DS_L1: Dicomitus squalis lectin 1 (Dicsq1); AO_L1: Arthrobotrys oligospora lectin 1 (s00075g2); LB_L1: Laccaria bicolor lectin 1 (Lbic_330799); LB_L2: Laccaria bicolor lectin 2 (Lbic_327918); MOA: Marasmius oreades agglutinin; SNA-II: Sambucus nigra agglutinin/ribosome inactivating protein type II. The distantly related canonical R-type lectins MOA (fungal, 14\% sequence identity) and SNA-II (plant, 13\% sequence identity) were included in the alignment based on comparison of their 3D structures [70,71]. The Clustal X color scheme was used. Residues involved in the carbohydrate recognition are indicated at the bottom for CCL2, MOA and SNA-II. The secondary structure of CCL2 and the conservation is indicated as well. The alignment was generated with Jalview [72]. doi:10.1371/journal.ppat.1002706.g006

multicellular fungi, in particular against predators and parasites [39]. Among the different types of potential fungal defence proteins identified $[13,26,40,41]$ the number and diversity of lectins is remarkably high, in accordance with the suitability of glycoepitopes for non-self recognition in innate defence mechanisms. Most fungal defence lectins are highly abundant in reproductive and long-term survival structures such as fruiting bodies and sclerotia, respectively, which require special protection
[13]. This expression pattern, also found for CGL1 and CCL2 (Figures 1B-C), is analogous to plants where the expression of many lectins is confined to seeds.

The strong and specific toxicity of CCL1 and CCL2 towards $D$. melanogaster and C. elegans is in accordance with the prevalence of the phyla Arthropoda and Nematoda as predators of mushrooms both in nature [42,43] and in mushroom farms [44,45]. In addition, this specificity of CCL1/CCL2-mediated toxicity corre- 

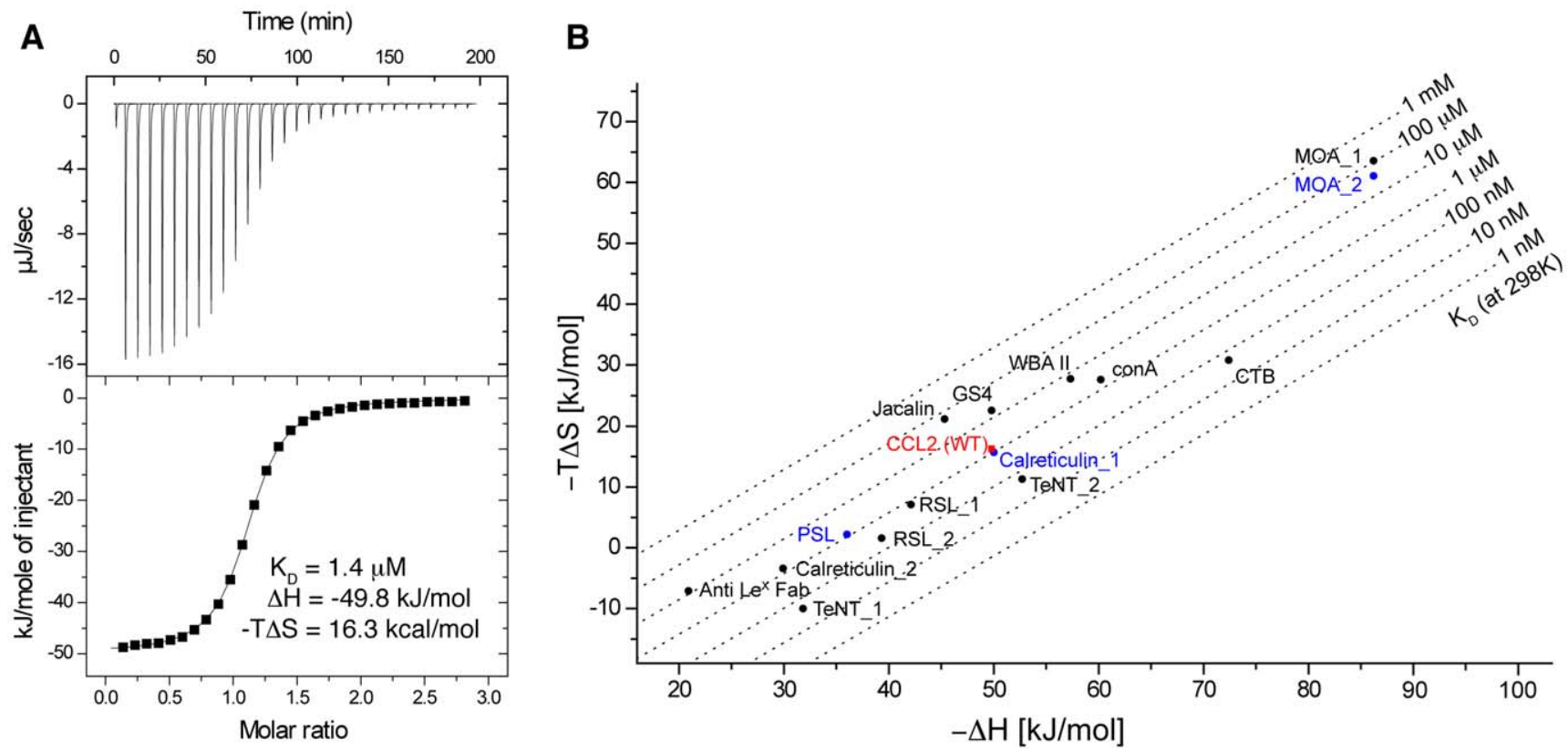

Figure 7. Thermodynamic binding parameters. (A) ITC experiment of wild type CCL2 binding to GlcNAc $\beta 1,4[\mathrm{Fuc} \alpha 1,3] \mathrm{GlcNAC}$-spacer. The raw calorimetric output is shown on top, the fitted binding isotherm at the bottom. The protein concentration in the cell was $70 \mu \mathrm{M}$, carbohydrate concentration in the syringe was $2.4 \mathrm{mM}$. (B) Thermodynamic binding parameters of CCL2 (in red) in comparison to other lectins with a focus on high affinity binding. Anti Le ${ }^{X}$ Fab: Fab fragment of the monoclonal antibody 291-2G3-4; ConA: concavalin A from jack bean seeds (Canavalia ensiformis); CTB: cholera-toxin B subdomain; GS4: Griffonia simplicifolia lectin 4; MOA: Marasmius oreades agglutinin; RSL: Ralstonia solanacearum fucose-binding lectin; TeNT: tetanus neurotoxin; WBA II: winged bean (Psophocarpus tetragonolobus) acidic agglutinin. For simplicity, lectins that use Ca ${ }^{2+}$ for carbohydrate recognition are not displayed. Details for each correlation are found in Table S6. Data points in blue are discussed in the text. doi:10.1371/journal.ppat.1002706.g007

lates with the identification of $\alpha 1,3$ fucosylated $\mathrm{N}$-glycan cores as target structures of these lectins in vivo (Figure 8), since this epitope is present exclusively in plant and invertebrate N-glycans [31]. The NMR structure revealed that CCL2 recognizes the fucosecontaining trisaccharide, GlcNAc $\beta 1,4[$ Fuc $\alpha 1,3]$ GlcNAc, as part of this epitope with high specificity. Within this trisaccharide, almost all functional groups of Fuc $\alpha 1,3 \mathrm{GlcNAc}$ and the acetamido group of the distal GlcNAc2 are recognized. The recognition of the distal saccharide is more relaxed, a GalNAc with an acetamido at the same position will be equally well recognized. Accordingly, among the glycans of the mammalian glycan array, GalNAc $\beta 1,4[\mathrm{Fu}-$ $c \alpha 1,3]$ GlcNAc (fucosylated LacdiNAc $=$ LDN-F) was one of the best binders. Since there is space for extensions at $\mathrm{O} 6$ of GlcNAcl and $\mathrm{O} 4$ of GlcNAc2 (Figure 5E) we can derive the following recognition sequence: $\mathrm{X}-1,4 \mathrm{GalNAc} / \mathrm{GlcNAc} \beta 1,4[\mathrm{Fuc} \alpha 1,3][\mathrm{Y}-$ $1,6]$ GlcNAc in which $\mathrm{X}$ and $\mathrm{Y}$ are tolerated extensions. In addition, binding of substituted Lewis ${ }^{\mathrm{X}}$ structures on the glycan array (Figure 2) suggests that substitutions at O3 and O6 of the galactose (corresponding to the distal GlcNAc in $\alpha 1,3$ fucosylated chitobiose) and at O6 of GlcNAc (corresponding to the proximal GlcNAc in $\alpha 1,3$ fucosylated chitobiose) are allowed. Accordingly, we would expect specific binding of CCL2 to paucimannose-type $\mathrm{N}$-glycans carrying both $\alpha 1,6$ and $\alpha 1,3$-linked fucose on the proximal and possibly $\alpha 1,3$-linked fucose on the distal GlcNAc (Figure 8A). The GlcNAc $\beta 1,4[$ Fuc $\alpha 1,3]$ GlcNAc motif is also a central part of the anti-HRP epitope that it is recognized by antisera raised against HRP in agreement with the isolation of CGL2 as HRP-binding lectin (Figure 1A). Since this epitope is also a key carbohydrate determinant of pollen and insect venom allergens [46], it appears that the same glycoepitope has been selected as target by the antibody-mediated mammalian adaptive immune system and a lectin-mediated fungal defence system.
The high affinity of CCL2 to the recognized trisaccharide determined by ITC is remarkable. Typically, individual carbohydrate binding sites of lectins have a rather low affinity to their ligands and this low affinity is usually compensated by multivalency achieved either by multiple binding sites on the same polypeptide chain or by oligomerization of polypeptide chains with one or few binding sites which leads to a high avidity towards multivalent ligands [47]. However, high affinity carbohydrate binding sites of lectins have been described and they differ from low affinity binding sites by their degree of specificity [48]: whereas low affinity binding sites often have a broad specificity towards terminal mono- or disaccharides present on many different glycans, high affinity sites recognize distinct oligosaccharides that are characteristic for specific glycans and glycoconjugates. The high affinity and specificity of the carbohydrate binding site in CGL2 towards the recognized trisaccharide is achieved by H-bonds and key hydrophobic contacts to almost all functional groups of Fuc $\alpha 1$,3GlcNAc as well as the acetamido group of the distal GlcNAc2. The ladder interaction is central for the high affinity, the absence of the distal acetamido group as in Gal $\beta 1,4[$ Fuc $\alpha 1,3]$ GlcNAc $\left(\right.$ Lewis $^{\mathrm{X}}$ ) leads to a drop in affinity by $\sim 300$ fold (in Table 1). To our knowledge, CCL2 is the only lectin that binds GlcNAc $\beta 1,4[$ Fuc $\alpha 1,3]$ GlcNAc with such a high specificity and affinity, making CCL2 superior to anti-HRP for detection of this glycoepitope. Since this and the other recognized glycoepitope, GalNAc $\beta 1,4[$ Fuc $\alpha 1,3]$ GlcNAc (LDN-F), are present in parasitic helminths [49-51], CCL2 may be used for the diagnostics of parasitic infections in animals and humans. The toxicity of CCL2-binding to at least one of these epitopes in vivo, may be exploited to develop novel approaches for the prevention or therapy of these infections. Another application could be the use of CCL2 on lectin microarrays for differential glycan profiling [52] or cellular glycomics [53]. 
A
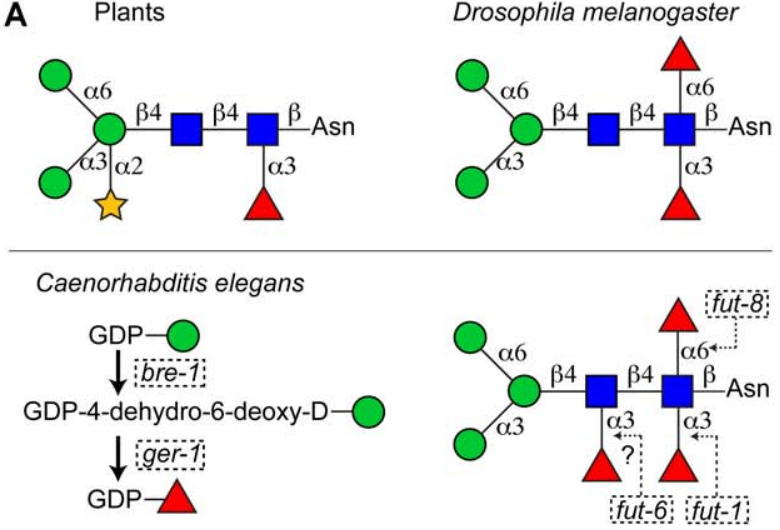

fut-6:

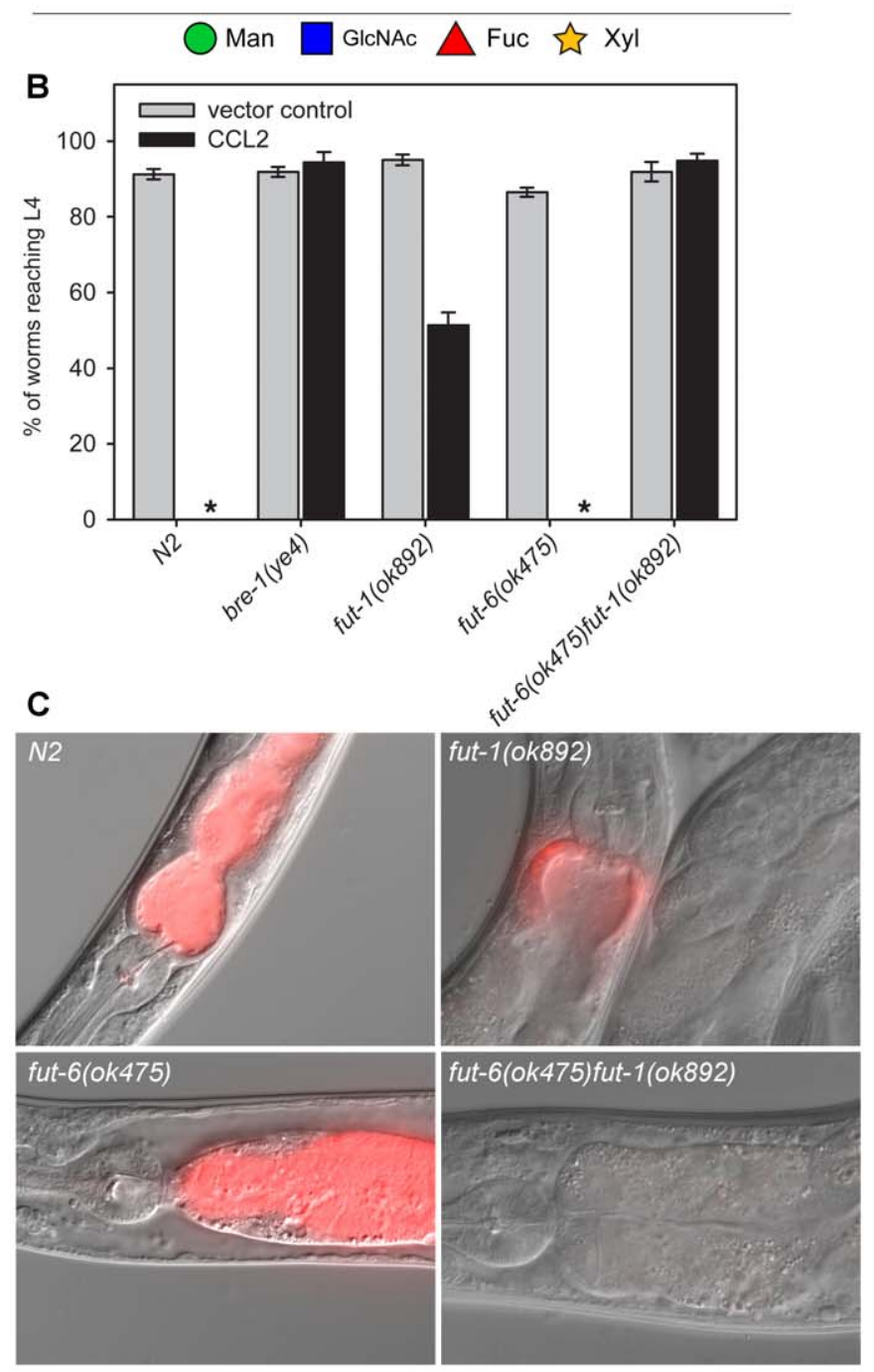

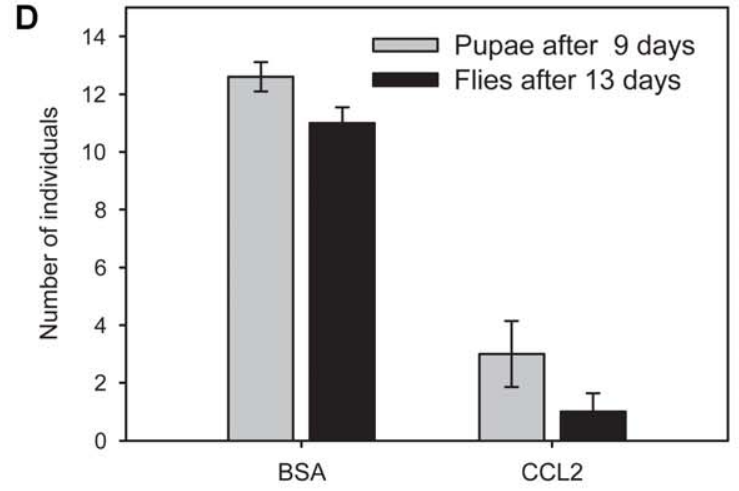

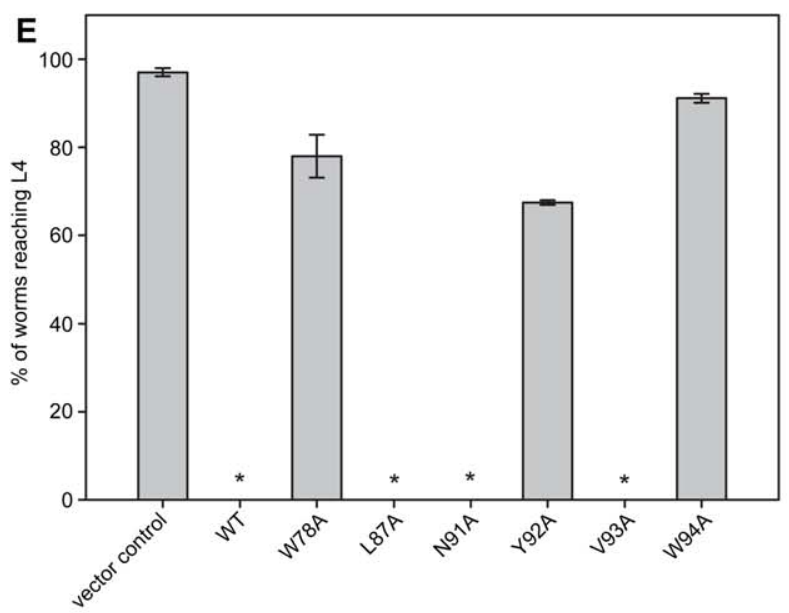

Figure 8. Carbohydrate-binding dependent biotoxicity of CCL2. (A) Schematic representation of N-glycan structures in plants, insects and nematodes. Upper panel, left: Typical paucimannosidic plant N-glycan, highly abundant in HRP. Upper panel right: Fucosylated paucimannosidic Nglycan present in D. melanogaster. Lower panel: Fucose biosynthesis and $\mathrm{N}$-glycan structure in C. elegans. Genes coding for enzymes involved in the fucose biosynthesis (lower panel, left) and fucose transfer to the core of $\mathrm{N}$-glycans in C. elegans (lower panel, right) are indicated in dashed boxes. (B) Toxicity of recombinant E. coli expressing CCL2 (black bars) towards C. elegans wildtype (N2) and various fucosylation mutants. Error bars indicate standard errors of the mean. Asterisks $\left(^{*}\right)$ show cases where all data were 0 . Significant differences were observed between the vector control and CCL2 for N2 ( $n=10, p=0.013)$, fut-1(ok892) $(n=10, p=0.013)$ and fut-6(ok475) $(n=10, p=0.013)$ worms, but not for bre-1(ye4) $(n=10, p=0.329)$ or fut6(ok475)fut-1(ok892) ( $n=10, p=0.329$ ). (C) Fluorescence microscopy of $C$. elegans feeding on $E$. coli expressing a dTomato-CCL2 fusion protein, showing the grinder and anterior part of the intestine. (D) Toxicity of purified CCL2 towards D. melanogaster quantified as number of developed pupae (gray bars) or flies (black bars). BSA was included as control. Error bars indicate standard errors of the mean. Development of pupae and flies treated with CCL2 were significantly different from the control (pupae: $n=10, p=0.013$; flies: $n=10, p=0.013$ ). (E) Toxicity of $E$. coli expressing 
different CCL2 variants with mutations in residues involved in carbohydrate binding towards C. elegans wildtype (N2). Vector control and CCL2 wildtype (WT) were included as controls. Asterisks $\left(^{*}\right)$ show cases where all data were 0 . Error bars indicate standard error of the mean. W78A, Y92A and W94A were significantly different from WT control $(n=10, p=0.013)$, whereas L87A, N91A, V93A were not $(n=10, p=1.0)$. doi:10.1371/journal.ppat.1002706.g008

The NMR solution structure of CGL2 in complex with its ligand demonstrates the versatility and plasticity of the $\beta$-trefoil fold with regard to carbohydrate binding. First, the carbohydrate specificity of CCL2 is very different from other $\beta$-trefoil lectins which recognize terminal galactose epitopes like Gal $\alpha 1,3 \mathrm{Gal}$ [19],

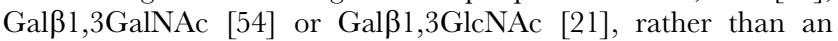
epitope with a terminal fucose. Second, unlike most $\beta$-trefoil lectins which utilize three almost identical binding sites per monomer, CCL2 recognizes the identified carbohydrate ligand via a single binding site. This binding site of CCL2 is located at a very unusual site of the $\beta$-trefoil fold, the interface between subdomains $\beta$ and $\gamma$. This stands in contrast to the fungal $\beta$-trefoil lectin SSA that also uses a single but canonical binding site [54]. None of the typical carbohydrate binding residues present in other $\beta$-trefoil lectins are found in CCL2 emphasizing the uniqueness of this non canonical binding site (Figure 6). Based on few $\beta$-trefoil complexes in which the binding site is slightly shifted from the canonical towards the CCL2 location [55-58] we speculate that this noncanonical binding site might have arisen from a previous recognition of other parts of the invertebrate $\mathrm{N}$-glycan by the canonical binding site $\beta$ (Figure $5 \mathrm{G}$ ) and then have changed to recognize another epitope of the same glycan by the non-canonical binding site. The key residues of the CCL2 binding site are highly conserved in CCL2 homologs of other fungi (Figure 6 and Table $\mathrm{S} 1$ ), but highly variable in other $\beta$-trefoil lectins. The unusual carbohydrate specificity is mainly based on $\mathrm{H}$-bonds from the protein main chain which requires the proper arrangement of three main chain sections: most importantly the characteristically short $\beta 7-\beta 8$ loop, strand $\beta 6$ and the $\beta 9-\beta 10$ loop. In particular, the short $\beta 7-\beta 8$ loop is conserved in all CCL2 homologues with a consensus sequence LPxxYVW, a signature we propose for the identification of lectins with a similar target specificity. In summary, based on sequence alignment we predict that the homologous CCL2 like genes of basidiomycetes have the same unusual binding location and the same target specificity as CGL2 (except LB_L2 that lacks the crucial Y93). As we do not have any evidence for a difference in regulation, specificity or function between the different paralogs, e.g. CGL1 and CCL2, we speculate that this redundancy is a strategy to avoid loss of specific defense effectors by individual gene mutations.

The strong toxicity of CGL2 towards $C$. elegans and $D$. melanogaster is surprising in the light of the monomeric state of the lectin in solution and the consequential lack of multivalency for the identified ligand since clustering of glycoconjugates on cell surfaces is generally regarded as a prerequisite for lectin-mediated toxicity [59]. CCL2 mutant proteins unable to bind the HRP epitope are not able to bind anymore to the C. elegans intestinal epithelium which rules out the presence of an additional binding site on CCL2 with different specificity for this tissue (A. Butschi, unpublished results). Thus, we hypothesize that the high affinity of the single carbohydrate-binding site of CCL2 compensates for the lack of multivalency and that CGL2 acts by a novel toxicity mechanism that does not seem to involve clustering. Accordingly, CCL2 variants with a lower affinity in vitro showed a reduced toxicity in C. elegans. Remarkably, the consequences of intoxication of $C$. elegans by $\mathrm{CCL1} / 2$ and the multivalent fruiting body lectins MOA and CGL2 are very similar, all of them leading to disintegration of the intestinal epithelium and a substantial enlargement of the intestinal lumen (Figure 8C) [10,16]. In addition, experiments aiming at the localization of the target glycoconjugates using fluorescently labeled CCL2 and CGL2 gave very similar results (Figure 8C) [16]. Interestingly, disintegration of the intestinal epithelium and enlargement of the intestinal lumen were also observed with the nematode-specific Cry toxins from Bacillus thuringiensis where carbohydrate-dependent binding to the intestinal epithelium appears to trigger expulsion of microvilli from the apical side of the intestinal epithelial cells [60]. In any case, interference with carbohydrate binding by the lectin, either by mutating genes involved in the biosynthesis of the identified target glycans in C. elegans or altering the identified carbohydrate binding sites in the lectin, abolished toxicity and binding of the fluorescently labeled lectin to the intestinal epithelium (Figure 8C) $[10,16]$. It should be noted, however, that not all variants of CCL2 were tested for toxicity towards C. elegans and none was tested for toxicity towards D. melanogaster. Thus, although we can show that the recognition of specific glycans is a crucial part of lectin-mediated defence mechanisms, the exact mechanisms of toxicity remain to be elucidated. Possible mechanisms are direct membrane damage or the interference with cellular signaling pathways, recycling of cell surface receptors, cell-cell or cell-matrix interactions. In order to distinguish between these possibilities and to find potential targets of novel antihelminthics, we are currently in the process of identifying the glycoprotein $(\mathrm{s})$ targeted by CGL2 and CGL2 in C. elegans.

\section{Materials and Methods}

\section{Carbohydrates}

Lewis $^{\mathrm{X}}$ trisaccharide methyl glycoside, $3^{\prime}$-Sialyl-Lewis ${ }^{\mathrm{X}}$ tetrasaccharide methyl glycoside and Fuc $\alpha 1,3 \mathrm{GlcNAc-OMe}$ were purchased from Carbosynth, UK. The chemically synthesized fucosylated chitobiose GlcNAc $\beta 1,4[\mathrm{Fuc} \alpha 1,3] \mathrm{GlcNAc} \beta-\mathrm{O}\left(\mathrm{CH}_{2}\right)_{5} \mathrm{COONa}$ [61] was a kind gift of Mayeul Collot, ENS, France. Lewis ${ }^{\mathrm{X}}$ tetrasaccharide and 3'-Sialyl-lactose were a kind gift of Eric Samain, GERMAV, France. The identity and purity of the carbohydrates was checked using 2D NMR spectroscopy.

\section{Strains and cultivation conditions}

Detailed information of the strains used in this study can be found in Table S7. Escherichia coli strain DH5 $\alpha$ was used for cloning and amplification of plasmids, strains BL21(DE3) and BL21(DE3)/pLysS were used for bacterial expression of proteins and biotoxicity assays and strain OP50 was used to feed C. elegans during regular breeding. Cultivation conditions of the various organisms are described in Text $\mathrm{S} 1$.

\section{Isolation and purification of CCL2 from C. cinerea}

CCL2 was isolated and purified from $C$. cinerea as described in Text S1.

\section{Identification of CCL2 by peptide mass fingerprinting}

Purified CCL2 was separated by SDS-PAGE, excised from the gel and identified by MALDI-MS/MS. Details of the procedure are described in Text $\mathrm{S} 1$.

\section{Quantification of $c c / 1$ and $c c / 2$ expression by qRT-PCR}

Details of the quantification are described in Text S1. 
Cloning of CCL1- and CCL2-encoding genes

The PCR-based cloning strategies for the various CGL1- and CCL2-encoding genes are described in Text S1.

\section{Determination of CCL1 and CCL2 expression levels in $C$. cinerea}

Protein expression of CCL2 was evaluated by immunoblotting. Soluble protein extracts of vegetative mycelium and fruiting bodies from C. cinerea were obtained as described above and separated on a $12 \%$ SDS-PAGE and probed with specific antiserum raised in rabbits against purified recombinant CCL2 (Pineda AntikörperSevice, Berlin, Germany) and detected with HRP-conjugated secondary antibodies. Transcription levels of both genes were assessed by quantitative real-time PCR (qRT-PCR) as described in Text S1.

\section{Glycan array analysis of CCL1 and CCL2}

Purified CGL1 and CCL2 were fluorescently labeled with Alexa Fluor 488 (Invitrogen) according to the manufacturer's protocol and used (at a final concentration of $200 \mu \mathrm{g} / \mathrm{ml}$ ) to probe versions 4.2 and 3.1, respectively, of the mammalian glycan array offered by Core $\mathrm{H}$ of the Consortium for Functional Glycomics (CFG).

\section{Preparation of proteins and their carbohydrate complexes}

Unlabelled and uniformly ${ }^{15} \mathrm{~N}$ or ${ }^{13} \mathrm{C} /{ }^{15} \mathrm{~N}$ labeled proteins were overexpressed in E. coli as His8-fusions and purified with affinity chromatography (see Text S1). Samples were dialyzed against NMR buffer (50 mM KH${ }_{2} \mathrm{PO}_{4}, \mathrm{pH} 5.7,150 \mathrm{mM} \mathrm{NaCl}$ ). Complexes of CCL2 with GlcNAc $\beta 1,4[$ Fuc $\alpha 1,3]$ GlcNAc $\beta$ $\mathrm{O}\left(\mathrm{CH}_{2}\right)_{5} \mathrm{COONa}$ were prepared by titrating the concentrated carbohydrate solution of typically $10 \mathrm{mM}$ into a $\sim 1 \mathrm{mM}$ solution of CCL2 in NMR buffer until a 1:1 stoichiometry was reached. Subsequently, the $\mathrm{pH}$ was lowered to 4.7 using $10 \%$ deuterated acetic acid to avoid precipitation.

\section{NMR spectroscopy}

NMR spectra were acquired on Avance III 500, 600, 700, 750 and Avance 900 Bruker spectrometers at $310 \mathrm{~K}$. NMR data were processed using Topspin 2.1 (Bruker) and analyzed with Sparky (Goddard, T.D. \& Kneller, D.G. SPARKY 3. University of California, San Francisco). The ${ }^{1} \mathrm{H},{ }^{13} \mathrm{C},{ }^{15} \mathrm{~N}$ chemical shifts of the protein, free and in complex, were assigned by standard methods [62]. Assignment of carbohydrate resonances of the complex was achieved using NOE correlations and exchange peaks with signals of the free carbohydrate since neither TOCSY based spectra nor a natural abundance ${ }^{13} \mathrm{C}$-HSQG showed bound carbohydrate signals. The following spectra were used for this purpose 2D ${ }^{1} \mathrm{H}-{ }^{1} \mathrm{H}$ NOESY, 2D ${ }^{13} \mathrm{C} /{ }^{15} \mathrm{~N}$ Fl-filtered NOESY and 2D ${ }^{13} \mathrm{C}$ F1filtered F2-filtered NOESY [63]. The assignments of intermolecular NOEs were derived from 3D ${ }^{13} \mathrm{C}$ F1-edited, F3-filtered NOESY-HSQC [27] spectra of the protein-carbohydrate complex. More details are found in the Text S1.

\section{Structure calculation and refinement}

The AtnosCandid software package [64,65] was used to generate initial CCL2 structures (free and bound) using three 3D NOESY spectra $\left({ }^{13} \mathrm{C}^{\text {ali }}\right.$-edited, ${ }^{13} \mathrm{C}^{\text {aro }}$-edited and ${ }^{15} \mathrm{~N}$-edited $)$ and one 2D NOESY spectrum. The automatically generated upper limit restraints file was used as a starting point for the first level of manually refining the protein structures by a simulated annealing protocol using the Cyana package [64]. Preliminary structures of the CCL2-carbohydrate complex were generated using the Cyana package with the above mentioned restraints and manually assigned intermolecular and intra-carbohydrate NOE distance constraints. To create the topology of the carbohydrate for the Cyana library file an initial model was generated by SWEET [66]. The carbohydrate spacer was truncated to a methyl group. 300 structures were generated by CYANA starting from random carbohydrate and protein starting structures. Ensemble of 30 structures of CCL2 free and in complex were refined with AMBER 9.0 [67].in implicit solvent using NOE-derived distances, torsion angles and hydrogen bond restraints as summarized in Table 2. For more details see Text S1. The Ramachandran statistics of CCL2 free and in complex, respectively, show $79.9 \%$ and $80.2 \%$ in the most favored regions, $18.0 \%$ and $18.7 \%$ in the additionally allowed regions, $1.5 \%$ and $1.0 \%$ in the generously allowed regions and $0.6 \%$ and $0.2 \%$ in the disallowed regions.

\section{Biotoxicity assays with recombinant $\mathrm{CCL} 2$}

Biotoxicity assays for $A$. aegypti and $A$. castellanii were performed with recombinant $E$. coli as previously described [30]. For $C$. elegans, a liquid toxicity assay was performed as follows: a synchronous population of L1 larvae as well as a bacterial culture of recombinant $E$. coli expressing CCL2 or containing a vector control were obtained as described [22]. E. coli cells were pelleted and re-suspended in sterile PBS to an $\mathrm{OD}_{600}=2$. The assay was set up in 96-well plates (TPP) by mixing $80 \mu \mathrm{l}$ of the bacterial suspension and $20 \mu \mathrm{l}$ of L1 larvae containing approximately 30 individuals. Each treatment (different bacterial and/or worm strain combinations) was done in 5 replicates. The worms were allowed to feed on the suspended bacteria at $20^{\circ} \mathrm{C}$ in the dark. The total number of animals and the percentage of individuals reaching L4 stage were quantified after $48 \mathrm{~h}$. The biotoxicity assay with $D$. melanogaster was performed adding purified protein to the rearing medium as previously described [68] using 20 eggs.

For the statistical analysis of the toxicity assays, pairwise comparisons were done using the non-parametric KolmogorovSmirnov test in the case of C. elegans, A. castellanii and D. melanogaster and the parametric T-student test for A. castellanii. The response variables (development, survival and clearing area) were compared between the tested lectin and the control or between mutant and wildtype.

\section{Preparation of the C. elegans fut- 1 fut- 6 double mutant (F1F6) and PCR screening}

Details are described in Text $\mathrm{S} 1$.

\section{Localization of CCL2-binding in C. elegans}

More information is found in Text S1.

\section{Isothermal titration calorimetry (ITC)}

ITC experiments were performed on a VP-ITC instrument (MicroCal). The calorimeter was calibrated according to the manufacturer's instructions. Protein and carbohydrate samples were dialyzed against NMR buffer at room temperature using a $3.5 \mathrm{kDa}$ membrane (Spectra/Por) and Micro DispoDialyzer (100 Da cutoff; Harvard Apparatus), respectively. The disaccharide Fuc $\alpha 1,3 \mathrm{GlcNAc-OMe}$ was not dialyzed but dissolved in NMR buffer. The sample cell $(1.4 \mathrm{~mL})$ was loaded with $70 \mu \mathrm{M}$ protein; carbohydrate concentration in the syringe was $2-4 \mathrm{mM}$. A titration experiment typically consisted of 30-50 injections, each of $3 \mu \mathrm{L}$ volume and $6 \mathrm{~s}$ duration, with a $6.7 \mathrm{~min}$ interval between additions. Stirring rate was $307 \mathrm{rpm}$. Raw data were integrated, corrected for nonspecific heats, normalized for the molar concentration, and analyzed according to a 1:1 binding model. 
Accession codes and numbers

The atomic coordinates of the structures of CCL2 free and in complex with the fucosylated chitobiose (GlcNAc $\beta 1,4[\mathrm{Fu}-$ $c \alpha 1,3]$ GlcNAc $\beta$-OMe) have been deposited in the Protein Data Bank with accession codes 2LIE and 2LIQ respectively. The chemical shifts of the free protein and in complex were deposited in the BioMagResBank (BMRB) under the accession numbers 17890 and 17902, respectively. The cDNA sequences of CCL1 and CCL2 from C. cinerea strain AmutBmut were deposited in GenBank under accession number ADO87036 and ACD88750, respectively.

\section{Supporting Information}

Figure S1 Coomassie-stained SDS-PAGE showing the expression and solubility of GCL1 and CGL2 recombinantly expressed in E.coli. WCE: whole cell extracts; SE: soluble fraction of WCE.

(TIF)

Figure S2 Size exclusion chromatography showing the monomeric state of GCL2 compared to standards proteins. (A) Elution profile of standard proteins (dotted lines, numbers indicate size in $\mathrm{kDa}$ ) and CCL2 (red) which elutes at $12.1 \mathrm{ml}$ from a Superdex 75 10/300 (GL) column. (B) Calibration curve done with the following standards: Ovalbumin $44 \mathrm{kDa}$, Carbonic anhydrase (29 kDa), Myoglobin (17 kDa), RNAse A (13.7 kDa) and Vitamin $\mathrm{Bl}_{2}(1.35 \mathrm{kDa})$. The void volume was determined by elution of bovine $\gamma$-globulin $(8.04 \mathrm{ml})$. The calculated molecular weight for CCL2 (in red) is 17.6. Gel filtration was performed at a flow rate of $0.5 \mathrm{ml} / \mathrm{min}$ in $10 \mathrm{mM}$ sodium phosphate, $150 \mathrm{mM} \mathrm{NaCl}$ buffer $\mathrm{pH}$ 6.2. Samples of $2-5 \mathrm{mg} / \mathrm{ml}$ of protein in $0.1 \mathrm{ml}$ were injected, and the eluate was monitored at $280 \mathrm{~nm}$.

(TIF)

Figure S3 Glycan array analysis showing the carbohydrate-binding specificity of CGL1. Results shown are averages of triplicate measurements of fluorescence intensity at a lectin concentration of $200 \mu \mathrm{g} / \mathrm{ml}$ probed on the Mammalian Glycan Array (V 4.2). Error bars indicate the standard deviations of the mean. Glycan structures are depicted for those epitopes with highest relative fluorescence. The raw data and the entire list of glycans with the respective spacers can be found on the CFG homepage [http://functionalglycomics.org/] or in Table S3. Binding of 6'sulfo-sialyllactose (glycan \#45) is likely to be an artifact since it is also bound by fucose-binding lectin AAL [http:/ / functionalglycomics.org/].

(TIF)

Figure S4 Isothermal titration calorimetry binding experiments between wild type CCL2 and different carbohydrate ligands. Raw calorimetric outputs are shown on the top and binding isotherms describing the complex formation are shown at the bottom. The protein concentration in the cell was $70 \mu \mathrm{M}$ and the carbohydrate concentration was $3.0 \mathrm{mM}$.

(TIF)

Figure S5 3D F1-edited F3-filtered HSQG-NOESY spectrum. A) Carbohydrate resonances are well dispersed in the direct dimension $\omega_{3}\left({ }^{13} \mathrm{C}\right.$ filtered/suppressed). Shown is a slice at the $\omega_{2}\left({ }^{13} \mathrm{C}\right.$ edited/selected $)$ resonance of V93 methyl group QG2 displaying intermolecular NOEs. B) Slice of the two indirect dimensions $\omega_{1}$ and $\omega_{2}$ at the $\omega_{3}$ resonance of Fucose $\mathrm{H} 2_{\text {bound }}$ showing intermolecular NOEs to Fucose $\mathrm{H} 2$. The ${ }^{1} \mathrm{H}-{ }^{13} \mathrm{C}$ correlations of the ${ }^{13} \mathrm{C}$ labelled protein were directly compared to the ${ }^{13} \mathrm{C}$ HSQG spectrum of the protein to assign the intermolecular NOEs.

(TIF)

Figure S6 The three symmetry-related canonical binding sites of $\beta$-trefoil proteins illustrated by the lectin MOA. (A-C) Three different side views related to each other by rotation of $120^{\circ}$ around the $\mathrm{z}$ axis of MOA in complex with Gal $\alpha 1,3$ (Fuc $\alpha 1,2)$ Gal (PDB: 3EF2). The binding sites are indicated by Greek letters. (D) Top view of the same complex. The same colors and similar orientations are used as for CCL2 in Figs. 4 and 5. (E) Superposition of the CGL2 complex structure (blue) with ligand (cyan) on the MOA complex structure 3EF2 (grey) with ligands (yellow). The $\beta$ subunit is located in front.

(TIF)

Figure S7 Angle plots of the glycosidic linkages of fucosylated chitobiose found in the 20 calculated complex structures. The plots, generated by CARP [73], display the observed angles (red with labels in blue) on top of an energy landscape calculated by modelling (top) or on top of angles of the same disaccharide linkage found in all structures deposited in the PDB database (bottom).

(TIF)

Figure S8 Toxicity of GCL2 towards $A$. aegypti and $A$. castellanii. Toxicity of CCL2-expressing $E$. coli towards larvae of the mosquito A. aegypti (A) and the amoebozoon $A$. castellanii $(\mathrm{B})$ was assessed as described in Materials and Methods. Error bars indicate standard errors of the mean. No significant differences were observed between CGL2 and VG ( $>>0.05)$.

(TIF)

Figure S9 Dose-dependence of GCL2-mediated nematotoxicity. Wildtype C. elegans (N2) were fed with mixtures of CCL2-expressing E. coli expressing CCL2 and empty vectorcontaining E. coli. Error bars indicate standard errors of the mean. (TIF)

Figure S10 Garbohydrate-binding dependence of GCL1mediated nematotoxicity. Toxicity of CCL1-expressing E. coli towards $C$. elegans wild type (N2) and various fucosylation mutants. Error bars indicate standard errors of the mean. Asterisks $\left(^{*}\right)$ show cases where all data were 0 . Assays were done in solid media as described [30].

(TIF)

Figure S11 Toxicity of recombinant $E$. coli expressing CCL2 towards $C$. elegans wildtype (N2) and various mutants in predicted or characterized fucosyltransferases (fut) or GlcNAc-transferases (gly). Assays were done in solid media as described [30]. Error bars indicate standard errors of the mean. Asterisks $\left(^{*}\right)$ show cases where all data were 0 . $\dagger$ : In the fut-1(ok892) mutants a partial resistance is observed. Although the larvae survive and develop, they require at least $24 \mathrm{~h}$ more to reach L4 and look thinner and paler than the complete resistant double mutant fut-6(ok475)fut-1(ok892).

(TIF)

Figure S12 All GCL2 proteins containing a point mutant are folded. ${ }^{15} \mathrm{~N}-\mathrm{HSQC}$ spectra of ${ }^{15} \mathrm{~N}$ labelled proteins. (TIF)

Figure S13 Isothermal titration calorimetry binding experiments between GGL2 mutants and fucosylated

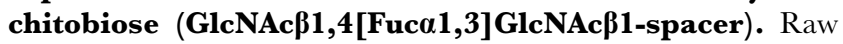
calorimetric outputs are shown on the top and binding isotherms describing the complex formation are shown at the bottom. The 
protein concentration in the cell was $70 \mu \mathrm{M}$ and the carbohydrate concentration was $3.0 \mathrm{mM}$.

(TIF)

Table S1 Sequence identities among the GCL2 homologues and the lectin MOA and SNA-II.

(PDF)

Table S2 Raw data of glycan array analysis performed with GCL2. RFU = Relative Fluorescence Units; SD = Standard deviation.

(PDF)

Table S3 Raw data of glycan array analysis performed with CGL1. RFU = Relative Fluorescence Units; SD = Standard deviation.

(PDF)

Table S4 Most prominent proteins structurally closest to GCL2 (free) obtained by a DALI search [74]. (PDF)

Table S5 Garbohydrate ${ }^{1} \mathrm{H}$ and ${ }^{13} \mathrm{G}$ chemical shifts [ppm] at $293 \mathrm{~K}$ referenced to DSS according to [75]. Bound chemical shifts were assigned via exchange peaks between the free and the bound form and by NOEs. Bound signals were not visible in a natural abundance ${ }^{13} \mathrm{C}$ HSQC.

(PDF)

Table S6 Thermodynamic data of selected lectin-carbohydrate interactions that are used in Fig. 7B.

(PDF)

Table S7 Strains used in this study.

(PDF)

\section{References}

1. Pilobello KT, Mahal LK (2007) Deciphering the glycocode: the complexity and analytical challenge of glycomics. Curr Opin Chem Biol 11: 300-305.

2. Marth JD, Grewal PK (2008) Mammalian glycosylation in immunity. Nat Rev Immunol 8: 874-887.

3. Hoffmann JA, Kafatos FC, Janeway CA, Ezekowitz RA (1999) Phylogenetic perspectives in innate immunity. Science 284: 1313-1318.

4. Vasta GR, Ahmed H, Tasumi S, Odom EW, Saito K (2007) Biological roles of lectins in innate immunity: molecular and structural basis for diversity in self/ non-self recognition. Adv Exp Med Biol 598: 389-406.

5. Stowell SR, Arthur CM, Dias-Baruffi M, Rodrigues LC, Gourdine JP, et al. (2010) Innate immune lectins kill bacteria expressing blood group antigen. Nat Med 16: 295-301.

6. Cash HL, Whitham CV, Behrendt CL, Hooper LV (2006) Symbiotic bacteria direct expression of an intestinal bactericidal lectin. Science 313: 1126-1130.

7. Stelter C, Kappeli R, Konig C, Krah A, Hardt WD, et al. (2011) Salmonellainduced mucosal lectin RegIIIbeta kills competing gut microbiota. PLoS One 6: e20749.

8. Kohatsu L, Hsu DK, Jegalian AG, Liu FT, Baum LG (2006) Galectin-3 induces death of Candida species expressing specific beta-1,2-linked mannans. J Immunol 177: 4718-4726.

9. Vandenborre G, Smagghe G, Van Damme EJ (2011) Plant lectins as defense proteins against phytophagous insects. Phytochemistry 72: 1538-1550.

10. Wohlschlager T, Butschi A, Zurfluh K, Vonesch SC, Auf dem Keller U, et al. (2011) Nematotoxicity of Marasmius Oreades Agglutinin (MOA) Depends on Glycolipid-Binding and Cysteine Protease Activity. J Biol Chem 286: 30337-30343.

11. Trigueros V, Lougarre A, Ali-Ahmed D, Rahbe Y, Guillot J, et al. (2003) Xerocomus chrysenteron lectin: identification of a new pesticidal protein. Biochim Biophys Acta 1621: 292-298.

12. Hamshou M, Van Damme EJ, Smagghe G (2010) Entomotoxic effects of fungal lectin from Rhizoctonia solani towards Spodoptera littoralis. Fungal Biol 114: 34- 40.

13. Bleuler-Martinez S, Butschi A, Garbani M, Wälti MA, Wohlschlager T, et al. (2011) A lectin-mediated resistance of higher fungi against predators and parasites. Mol Ecol 20: 3056-3070.

14. Goldstein IJ, Winter HC (2007) Mushroom Lectins. In: Kamerling JP, ed. Comprehensive Glycoscience: From Chemistry to Systems biology. Amsterdam: Elsevier Ltd. pp 601-621.

15. Hamshou M, Smagghe G, Shahidi-Noghabi S, De Geyter E, Lannoo N, et al. (2010) Insecticidal properties of Sclerotinia sclerotiorum agglutinin and its interaction with insect tissues and cells. Insect Biochem Mol Biol 40: 883-890.
Table S8 Oligonucleotides used in this study. Restriction sites in the oligonucleotides are underlined, and codon changes for site directed mutagenesis are in bold.

(PDF)

\section{Text S1 Supplementary methods.}

(PDF)

\section{Acknowledgments}

We thank Yuehan Feng, Sonja Käser and Yannick Duport for excellent technical support. We thank Mayeul Collot (Ecole Normale Superieure, CNRS, Paris, France) and Eric Samain (CERMAV, Grenoble, France) for providing oligosaccharides. We thank W. Rudin and P. Müller (Swiss Tropical and Public Health Institute, Basel, Switzerland) and E. Hafen and H. Stocker (Institute of Molecular Systems Biology, ETH Zürich, Switzerland) for the supply with $A$. aegypti and D. melanogaster eggs, respectively. We are grateful to Verena Jantsch (University of Vienna, Austria) for advice in preparing the C. elegans fut-6(ok475)fut-1(ok892) double mutant, to Markus Blatter for help with the structure calculation, especially the parameterization of the sugars, and to Julien Boudet for his introduction into the practical aspects of ITC measurements. We thank Helena Kovacs and Rainer Kümmerle for measuring with us several spectra at Bruker in Fällanden and for helpful discussions. We are grateful to Robert Woods for providing the GLYCAM tools and to Rudolf Glockshuber and Vikram Panse for helpful discussions.

\section{Author Contributions}

Conceived and designed the experiments: MS MK SBM MAW IBHW MOH MA FHTA. Performed the experiments: MS SBM AB MAW PE KS SY. Analyzed the data: MS SBM AB. Contributed reagents/materials/ analysis tools: SY. Wrote the paper: MS SBM FHTA MK.

16. Butschi A, Titz A, Walti MA, Olieric V, Paschinger K, et al. (2010) Caenorhabditis elegans $\mathrm{N}$-glycan core beta-galactoside confers sensitivity towards nematotoxic fungal galectin CGL2. PLoS Pathog 6: e1000717.

17. Lehotzky RE, Partch CL, Mukherjee S, Cash HL, Goldman WE, et al. (2010) Molecular basis for peptidoglycan recognition by a bactericidal lectin. Proc Natl Acad Sci U S A 107: 7722-7727.

18. Cummings RD, Etzler ME (2009) R-type Lectins. In: Varki A, Cummings RD, Esko JD, Freeze HH, Stanley P et al, eds. Essentials of Glycobiology. 2nd ed. Cold Spring Harbor (NY): Cold Spring Harbor Laboratory Press. pp 403-414.

19. Grahn EM, Winter HC, Tateno H, Goldstein IJ, Krengel U (2009) Structural characterization of a lectin from the mushroom Marasmius oreades in complex with the blood group B trisaccharide and calcium. J Mol Biol 390: 457-466.

20. Kadirvelraj R, Grant OC, Goldstein IJ, Winter HC, Tateno H, et al. (2011) Structure and binding analysis of Polyporus squamosus lectin in complex with the Neu5Ac\{alpha\}2-6Gal\{beta\}1-4GlcNAc human-type influenza receptor. Glycobiology 21: 973-984.

21. Mancheno JM, Tateno H, Goldstein IJ, Martinez-Ripoll M, Hermoso JA (2005) Structural analysis of the Laetiporus sulphureus hemolytic pore-forming lectin in complex with sugars. J Biol Chem 280: 17251-17259.

22. Pohleven J, Renko M, Magister S, Smith DF, Künzler M, et al. (2012) Bivalent carbohydrate-binding is required for biological activity of CNL, the LacdiNAc (GalNAcb1-4GlcNAc)-specific lectin from basidiomycete Clitocybe nebularis. J Biol Chem 287: 10602-10612.

23. Boulianne RP, Liu Y, Aebi M, Lu BC, Kues U (2000) Fruiting body development in Coprinus cinereus: regulated expression of two galectins secreted by a non-classical pathway. Microbiology 146(Pt 8): 1841-1853.

24. Wälti MA, Walser PJ, Thore S, Grunler A, Bednar M, et al. (2008) Structural basis for chitotetraose coordination by CGL3, a novel galectin-related protein from Coprinopsis cinerea. J Mol Biol 379: 146-159.

25. Hazes B (1996) The (QxW)3 domain: a flexible lectin scaffold. Protein Sci 5: 1490-1501.

26. Renko M, Sabotic J, Mihelic M, Brzin J, Kos J, et al. (2010) Versatile loops in mycocypins inhibit three protease families. J Biol Chem 285: 308-316.

27. Dominguez C, Schubert M, Duss O, Ravindranathan S, Allain FH (2011) Structure determination and dynamics of protein-RNA complexes by NMR spectroscopy. Prog Nucl Magn Reson Spectrosc 58: 1-61.

28. Kozlov G, Pocanschi CL, Rosenauer A, Bastos-Aristizabal S, Gorelik A, et al. (2010) Structural basis of carbohydrate recognition by calreticulin. J Biol Chem 285: 38612-38620. 
29. Tateno H, Winter HC, Goldstein IJ (2004) Cloning, expression in Escherichia coli and characterization of the recombinant Neu5Acalpha2,6Galbeta1,4GlcNAc-specific high-affinity lectin and its mutants from the mushroom Polyporus squamosus. Biochem J 382: 667-675.

30. Künzler M, Bleuler-Martinez S, Butschi A, Garbani M, Luthy P, et al. (2010) Biotoxicity assays for fruiting body lectins and other cytoplasmic proteins. Methods Enzymol 480: 141-150.

31. Paschinger K, Rendic D, Wilson IB (2009) Revealing the anti-HRP epitope in Drosophila and Caenorhabditis. Glycoconj J 26: 385-395.

32. Paschinger K, Rendic D, Lochnit G, Jantsch V, Wilson IB (2004) Molecular basis of anti-horseradish peroxidase staining in Caenorhabditis elegans. J Biol Chem 279: 49588-49598.

33. Haslam SM, Coles GC, Munn EA, Smith TS, Smith HF, et al. (1996) Haemonchus contortus glycoproteins contain N-linked oligosaccharides with novel highly fucosylated core structures. J Biol Chem 271: 30561-30570.

34. Vinck A, de Bekker C, Ossin A, Ohm RA, de Vries RP, et al. (2011) Heterogenic expression of genes encoding secreted proteins at the periphery of Aspergillus niger colonies. Environ Microbiol 13: 216-225.

35. Peumans WJ, Van Damme EJ (1995) Lectins as plant defense proteins. Plant Physiol 109: 347-352.

36. Chen MS (2008) Inducible direct plant defense against insect herbivores: A review. Insect Sci 15: 101-114.

37. Kessler A, Baldwin IT (2002) Plant responses to insect herbivory: the emerging molecular analysis. Annu Rev Plant Biol 53: 299-328.

38. Spiteller P (2008) Chemical defence strategies of higher fungi. Chemistry 14: 9100-9110

39. Wang M, Trigueros V, Paquereau L, Chavant L, Fournier D (2002) Proteins as active compounds involved in insecticidal activity of mushroom fruitbodies. J Econ Entomol 95: 603-607.

40. Lacadena J, Alvarez-Garcia E, Carreras-Sangra N, Herrero-Galan E, AlegreCebollada J, et al. (2007) Fungal ribotoxins: molecular dissection of a family of natural killers. FEMS Microbiol Rev 31: 212-237.

41. Berne S, Lah L, Sepcic K (2009) Aegerolysins: structure, function, and putative biological role. Protein Sci 18: 694-706.

42. McGonigle TP (2007) Effects of Animals Grazing Fungi. In: Kubicek CP, ed. Environmental and Microbial Relationships. Berlin Heidelberg: SpringerVerlag. pp 201-212.

43. Ruess L, Lussenhop J (2005) Trophic interactions of Fungi and Animals. In: Dighton J, White JF, Oudemans P, eds. The Fungal Community Its Organization and Role in the Ecosystems. Boca Raton: CRC Press. pp 581-598.

44. Shamshad A (2010) The development of integrated pest management for the control of mushroom sciarid flies, Lycoriella ingenua (Dufour) and Bradysia ocellaris (Comstock), in cultivated mushrooms. Pest Manag Sci 66: 1063-1074.

45. Sanchez C (2010) Cultivation of Pleurotus ostreatus and other edible mushrooms. Appl Microbiol Biotechnol 85: 1321-1337.

46. Hoffmann-Sommergruber K, Paschinger K, Wilson IB (2011) Glycomarkers in parasitic infections and allergy. Biochem Soc Trans 39: 360-364.

47. Dam TK, Brewer CF (2010) Lectins as pattern recognition molecules: the effects of epitope density in innate immunity. Glycobiology 20: 270-279.

48. Imberty A, Mitchell EP, Wimmerova M (2005) Structural basis of high-affinity glycan recognition by bacterial and fungal lectins. Curr Opin Struct Biol 15: $525-534$.

49. van Die I, Gomord V, Kooyman FN, van den Berg TK, Cummings RD, et al. (1999) Core alphal $\rightarrow 3$-fucose is a common modification of N-glycans in parasitic helminths and constitutes an important epitope for IgE from Haemonchus contortus infected sheep. FEBS Lett 463: 189-193.

50. Aranzamendi C, Tefsen B, Jansen M, Chiumiento L, Bruschi F, et al. (2011) Glycan microarray profiling of parasite infection sera identifies the LDNF glycan as a potential antigen for serodiagnosis of trichinellosis. Exp Parasitol 129: 221-226.

51. Wuhrer M, Koeleman CA, Fitzpatrick JM, Hoffmann KF, Deelder AM, et al. (2006) Gender-specific expression of complex-type N-glycans in schistosomes. Glycobiology 16: 991-1006.

52. Kuno A, Matsuda A, Ikehara Y, Narimatsu H, Hirabayashi J (2010) Differential glycan profiling by lectin microarray targeting tissue specimens. Methods Enzymol 478: 165-179.

53. Tateno H, Kuno A, Itakura Y, Hirabayashi J (2010) A versatile technology for cellular glycomics using lectin microarray. Methods Enzymol 478: 181-195.
54. Sulzenbacher G, Roig-Zamboni V, Peumans WJ, Rouge P, Van Damme EJ, et al. (2010) Crystal structure of the GalNAc/Gal-specific agglutinin from the phytopathogenic ascomycete Sclerotinia sclerotiorum reveals novel adaptation of a beta-trefoil domain. J Mol Biol 400: 715-723.

55. Miyanaga A, Koseki T, Matsuzawa H, Wakagi T, Shoun H, et al. (2004) Crystal structure of a family 54 alpha-L-arabinofuranosidase reveals a novel carbohydrate-binding module that can bind arabinose. J Biol Chem 279: 44907-44914.

56. Strotmeier J, Gu S, Jutzi S, Mahrhold S, Zhou J, et al. (2011) The biological activity of botulinum neurotoxin type $\mathrm{C}$ is dependent upon novel types of ganglioside binding sites. Mol Microbiol 81: 143-156.

57. Liu Y, Misulovin Z, Bjorkman PJ (2001) The molecular mechanism of sulfated carbohydrate recognition by the cysteine-rich domain of mannose receptor. J Mol Biol 305: 481-490.

58. Faham S, Hileman RE, Fromm JR, Linhardt RJ, Rees DC (1996) Heparin structure and interactions with basic fibroblast growth factor. Science 271 : $1116-1120$.

59. Rabinovich GA, Toscano MA, Jackson SS, Vasta GR (2007) Functions of cell surface galectin-glycoprotein lattices. Curr Opin Struct Biol 17: 513-520.

60. Los FC, Kao CY, Smitham J, McDonald KL, Ha C, et al. (2011) RAB-5- and RAB-11-dependent vesicle-trafficking pathways are required for plasma membrane repair after attack by bacterial pore-forming toxin. Cell Host Microbe 9: 147-157.

61. Collot M, Wilson IB, Bublin M, Hoffmann-Sommergruber K, Mallet JM (2011) Synthesis of cross-reactive carbohydrate determinants fragments as tools for in vitro allergy diagnosis. Bioorg Med Chem 19: 1306-1320.

62. Sattler M, Schleucher J, Griesinger C (1999) Heteronuclear multidimensional NMR experiments for the structure determination of proteins in solution employing pulsed field gradients. Prog Nucl Magn Reson Spectrosc 34: 93-158.

63. Peterson RD, Theimer CA, Wu H, Feigon J (2004) New applications of 2D filtered/edited NOESY for assignment and structure elucidation of RNA and RNA-protein complexes. J Biomol NMR 28: 59-67.

64. Herrmann T, Guntert P, Wuthrich K (2002) Protein NMR structure determination with automated NOE assignment using the new software CANDID and the torsion angle dynamics algorithm DYANA. J Mol Biol 319: 209-227.

65. Herrmann T, Guntert P, Wuthrich K (2002) Protein NMR structure determination with automated NOE-identification in the NOESY spectra using the new software ATNOS. J Biomol NMR 24: 171-189.

66. Bohne A, Lang E, von der Lieth CW (1999) SWEET - WWW-based rapid 3D construction of oligo- and polysaccharides. Bioinformatics 15: 767-768.

67. Case DA, Cheatham TE, Darden T, Gohlke H, Luo R, et al. (2005) The Amber biomolecular simulation programs. J Comput Chem 26: 1668-1688.

68. Sabotic J, Bleuler-Martinez S, Renko M, Avanzo Caglic P, Kallert S, et al (2012) Structural basis of trypsin inhibition and entomotoxicity of cospin, a serine protease inhibitor involved in defence of Coprinopsis cinerea fruiting bodies. J Biol Chem 287: 3898-3907.

69. Taylor ME, Drickamer K (2006) Introduction to Glycobiology. Oxford: Oxford University Press. 160 p.

70. Grahn E, Askarieh G, Holmner A, Tateno H, Winter HC, et al. (2007) Crystal structure of the Marasmius oreades mushroom lectin in complex with a xenotransplantation epitope. J Mol Biol 369: 710-721.

71. Maveyraud L, Niwa H, Guillet V, Svergun DI, Konarev PV, et al. (2009) Structural basis for sugar recognition, including the Tn carcinoma antigen, by the lectin SNA-II from Sambucus nigra. Proteins 75: 89-103.

72. Waterhouse AM, Procter JB, Martin DM, Clamp M, Barton GJ (2009) Jalview Version 2-a multiple sequence alignment editor and analysis workbench. Bioinformatics 25: 1189-1191.

73. Lütteke T, Frank M, von der Lieth CW (2005) Carbohydrate Structure Suite (CSS): analysis of carbohydrate 3D structures derived from the PDB. Nucleic Acids Res 33: D242-246.

74. Holm L, Sander C (1995) Dali: a network tool for protein structure comparison. Trends Biochem Sci 20: 478-480.

75. Markley JL, Bax A, Arata Y, Hilbers CW, Kaptein R, et al. (1998) Recommendations for the presentation of NMR structures of proteins and nucleic acids. IUPAC-IUBMB-IUPAB Inter-Union Task Group on the Standardization of Data Bases of Protein and Nucleic Acid Structures Determined by NMR Spectroscopy. J Biomol NMR 12: 1-23. 